\title{
Branching process associated with 2d-Navier Stokes equation
}

\author{
Saïd Benachour, Bernard Roynette and Pierre Vallois
}

\begin{abstract}
Omega$ being a bounded open set in $\mathbb{R}^{2}$, with regular boundary, we associate with Navier-Stokes equation in $\Omega$ where the velocity is null on $\partial \Omega$, a non-linear branching process $\left(Y_{t} ; t \geq 0\right)$. More precisely: $E_{\omega_{0}}\left(\left\langle h, Y_{t}\right\rangle\right)=\langle\omega, h\rangle$, for any test function $h$, where $\omega=\operatorname{rot} u$, $u$ denotes the velocity solution of Navier-Stokes equation. The support of the random measure $Y_{t}$ increases or decreases of one unit when the underlying process hits $\partial \Omega$; this stochastic phenomenon corresponds to the creation-annihilation of vortex localized at the boundary of $\Omega$.
\end{abstract}

\section{Introduction.}

We consider here the $2 \mathrm{~d}-\mathrm{Navier-Stokes}$ (N.S.) equation in a bounded open set $\Omega$ of $\mathbb{R}^{2}$. The aim is not the study of existence or uniqueness for the solution, when the initial data or the boundary of $\Omega$ are smooth enough. We suppose that there exists a unique smooth solution $u$ of the Navier-Stokes equation, and we represent $u$ and the vorticity $(\omega=\operatorname{rot} u)$ through a stochastic model.

We firstly introduce two dual nonlinear differential systems. We prove (see sections 2 and 3 ) that the (N.S.) equation is equivalent to each of the former nonlinear reflected stochastic differential equations. The nonlinear feature appears in two places:

- inside $\Omega$, through the singular kernel of Biot and Savart, defining the mean velocity of the stochastic particle, when it moves in $\Omega$, 
- on the boundary of $\Omega$, via the local time; this process governing the local behaviour of the particle when it reaches $\partial \Omega$.

Since $\Omega$ is a subset of $\mathbb{R}^{2}$, the vorticity $\omega$ is a scalar function. Roughly speaking $\omega(t, \cdot)$ is the "density" of one of the two previous diffusion processes taken a time $t$. (Corollary 3.4).

Secondly we define a branching process $Y$, having again a double non linearity. By definition, $Y_{t}$ is a linear and random combination of Dirac measures. $\omega$ is expressed through $Y$ as follows ( $c f$. Theorem 4.5)

$$
E\left[\left\langle h, Y_{t}\right\rangle\right]=E\left[\int_{\bar{\Omega}} h(x) Y_{t}(d x)\right]=\int_{\Omega} h(x) \omega(t, x) d x,
$$

$h$ being a test function.

$\omega(t, x) d x$ can be interpreted as the mean value at time $t$ of the number of particles associated with $Y$, lying in a infinitesimal box located at $x$, with area $d x$. We heuristically describe the dynamic of branching of $Y$ :

- a single particle moves in $\Omega$ as a diffusion process introduced in the first step, all the particles being alive are independent,

- no particle is created when all of them lie in $\Omega$.

- Sometime (i.e. randomly), when a particle hits $F \subset \partial \Omega$ (respectively $F^{\dagger} \subset \partial \Omega$ ) the particle dies and give rise to two new independent particles, (resp. the particle dies), where $F \cup F^{\dagger}=\partial \Omega$. The branching mechanism taking on the boundary gives a stochastic interpretation of the creation or disappearing of vorticity on $\partial \Omega$.

We conjecture that the nonlinear branching process can be approximated by a system of interacting particles. Our algorithm does not coincide with those intoduced by A. Chorin ([C.M $]$ ).

Let in briefly detail the organisation of the paper:

- In Section 1, we study the connections between the Navier-Stokes equation and the equation verified by the vorticity $\omega$.

- In sections 2 and 3, we prove the equivalence between the two reflected stochastic differential equations and the (N.S.) equation. Moreover we check that these diffusion processes are in duality.

- We detail in Section 4, the construction of the branching process associated with the (N.S.) equation.

- We describe in Section 5, our open question concerning the simulation of the nonlinear branching process through a system of particles. 


\section{A first approach to the Navier Stokes equation.}

1) In this paper, $\Omega$ will denote a simply connected, bounded open subset of $\mathbb{R}^{2}$. We assume that the boundary $\partial \Omega$ is smooth. The Navier Stokes system in $\Omega$, with velocity vanishing at $\partial \Omega$, is

$$
\left\{\begin{array}{l}
\text { i) } \frac{\partial u}{\partial t}+(u \cdot \nabla) u=\nu \Delta u-\nabla p, \\
\text { ii) } \operatorname{div} u=0, \\
\text { iii) } u(0, \cdot)=u_{0}(\cdot), \\
\text { iv) } u(t, x)=0, \quad \text { for all } t \geq 0, \text { for all } x \in \partial \Omega .
\end{array}\right.
$$

$u=\left(u_{1}, u_{2}\right)$ is the velocity ( $u$ is a two-dimensional valued vector field), $u_{0}$ is the initial velocity, $p$ denotes the pressure ( $p$ is a scalar function), $\nu$ is the viscosity of the fluid ( $\nu$ will be taken without loss of generality equal to $1 / 2$ in the sequel). As usual

$$
\begin{gathered}
\nabla p=\left(\frac{\partial p}{\partial x}, \frac{\partial p}{\partial y}\right), \\
\operatorname{div} u=\frac{\partial u_{1}}{\partial x}+\frac{\partial u_{2}}{\partial y} \\
\Delta u=\frac{\partial^{2} u}{\partial x^{2}}+\frac{\partial^{2} u}{\partial y^{2}}
\end{gathered}
$$

and

$$
u \cdot \nabla=u_{1} \frac{\partial}{\partial x}+u_{2} \frac{\partial}{\partial y} .
$$

Note that the first equation i) in the (N.S.) system has to be understood as an equation in $\mathbb{R}^{2}$. Condition ii), i.e. $\operatorname{div} u=0$, means that the fluid is incompressible.

We know that, if $u_{0}$ with $\operatorname{div} u_{0}=0$ and $\partial \Omega$ are smooth, then (N.S.) has a unique smooth solution defined on $\mathbb{R}^{+} \times \bar{\Omega}$ ([La], [Li], [C-F]) if $u_{0}$ is $C^{\infty}(\bar{\Omega})$ and $([\mathrm{Ka}],[\mathrm{Ko}])$ if $u_{0}$ is analytic.

2) In a first step we weaken iv) and consider

$(\text { N.S. })^{\prime} \quad\left\{\begin{array}{l}\text { i) } \frac{\partial u}{\partial t}+(u \cdot \nabla) u=\frac{1}{2} \Delta u-\nabla p, \\ \text { ii) } \operatorname{div} u=0, \\ \text { iii) } u(0, \cdot)=u_{0}, \\ \text { iv) } u \cdot n(t, x)=0, \quad \text { for all } t \geq 0, \text { for all } x \in \partial \Omega .\end{array}\right.$ 
where $n(x)$ denotes the normalized outer normal vector at $x \in \partial \Omega$. Since iv) in (N.S.) is stronger than iv) in (N.S.) $)^{\prime}$ the solutions of (N.S.)' are not unique.

If $w: \mathbb{R}^{2} \longrightarrow \mathbb{R}$ we set

$$
\nabla^{\perp} w=\left(-\frac{\partial w}{\partial y},+\frac{\partial w}{\partial x}\right)
$$

Let us introduce the following operator $K$ ( $K$ is the Biot and Savart kernel associated with $\Omega$ )

$$
\begin{aligned}
K f(t, z)= & \nabla_{z}^{\perp} \int_{\Omega} G\left(z, z^{\prime}\right) f\left(t, z^{\prime}\right) d z^{\prime}, \\
= & \left\{\begin{array}{l}
-\frac{\partial}{\partial y} \int_{\Omega} G\left((x, y), z^{\prime}\right) f\left(t, z^{\prime}\right) d z^{\prime} \\
+\frac{\partial}{\partial x} \int_{\Omega} G\left((x, y), z^{\prime}\right) f\left(t, z^{\prime}\right) d z^{\prime}
\end{array}\right.
\end{aligned}
$$

where $z=(x, y)$ and $G$ is the Green function of $\Delta$ on $\Omega$, i.e.

$\Delta_{z^{\prime}} G\left(z, z^{\prime}\right)=\delta_{z}\left(\delta_{z}\right.$ being the Dirac measure at $\left.z\right)$,

$$
\begin{gathered}
G\left(z, z^{\prime}\right)=0, \quad \text { if } z^{\prime} \in \partial \Omega . \\
G\left(z, z^{\prime}\right)=G\left(z^{\prime}, z\right) .
\end{gathered}
$$

It is classical to replace the two-dimensional equation i) in (N.S.) (or $\left.(\text { N.S. })^{\prime}\right)$ by an equivalent equation where the unknown parameter will be a real function $\omega$. $\omega$ is called the vorticity associated with $u$ and is defined by

$$
\omega=\operatorname{rot} u:=\frac{\partial u_{2}}{\partial x}-\frac{\partial u_{1}}{\partial y} .
$$

Recall that it is supposed that $\Omega$ is a bounded simply connected open set.

Lemma 1.1. 1) Suppose $u: \bar{\Omega} \longrightarrow \mathbb{R}^{2}$ being a smooth function i) and ii) below are equivalent

i) $\operatorname{div} u=0$ in $\bar{\Omega}, u \cdot n=0$ in $\partial \Omega$.

ii) There exists $\omega: \bar{\Omega} \longrightarrow \mathbb{R}$ such that $u=K \omega$. 
In this case $\omega=\operatorname{rot} u$.

2) a) Assume $(u, p)$ is a solution of (N.S.)', then $\omega=\operatorname{rot} u$ solves the vorticity equation

$$
\left\{\begin{array}{l}
\text { i) } \left.\frac{\partial \omega}{\partial t}=\frac{1}{2} \Delta \omega-K \omega \cdot \nabla \omega, \text { in }\right] 0,+\infty[\times \Omega, \\
\text { ii) } \omega(0, \cdot)=\operatorname{rot} u_{0}:=\omega_{0} .
\end{array}\right.
$$

b) Suppose that $\omega$ is a solution of (1.8); then there exists $p$ such that $(u, p)$ solves (N.S.)' where $u=K \omega$.

ProOF. 1) $\Omega$ being a simply connected open set, the condition $\operatorname{div} u=0$ implies the existence of a function $\psi$ (the stream function) such that,

$$
u=\nabla^{\perp} \psi .
$$

Obviously $\psi$ is defined up to an additive constant. If we take a parametrization of $\partial \Omega$, we easily verify

$$
u \cdot n(t, x)=0, \quad \text { for all } \mathrm{t} \geq 0, \text { for all } x \in \partial \Omega
$$

if and only if $\psi(t, x)=c, \quad$ for all $t \geq 0$, for all $x \in \partial \Omega$.

$\psi$ is unique if we choose $c=0$.

We set $\omega=\operatorname{rot} u$. By a straightforward calculation we obtain

$$
\omega=\Delta \psi
$$

Since $\psi$ vanishes on $\partial \Omega$, it can be expressed through $\omega$, via the Green function

$$
\psi(t, z)=\int_{\Omega} G\left(z, z^{\prime}\right) \omega\left(t, z^{\prime}\right) d z^{\prime}
$$

(1.9) implies that $u=K \omega$.

We now analyze the converse. Suppose that $u=K \omega$. This means that $u=\nabla^{\perp} \psi$ when $\psi$ is defined by (1.12). Hence, $\operatorname{div} u=0$ in $\left[0,+\infty\left[\times \Omega\right.\right.$ and $\psi(t, x)=0$ for any $(t, x) \in \mathbb{R}_{+} \times \partial \Omega$. Then the equivalence (1.10) implies that $u \cdot n=0$.

2) a) For any smooth functions, $w: \mathbb{R}^{2} \longrightarrow \mathbb{R}^{2}$, and $q: \mathbb{R}^{2} \longrightarrow \mathbb{R}$, we have,

$(1.13) \operatorname{rot}(\nabla q)=0, \quad \operatorname{rot}((w \cdot \nabla) w)=w \cdot \nabla(\operatorname{rot} w)+(\operatorname{div} w) \operatorname{rot} w$ 
Let $(u, p)$ be a solution of (N.S. $)^{\prime}$. We take the rotational operator on both sides of (N.S.)' i), we easily obtain (1.8) i).

b) Let us suppose that $\omega$ solves (1.8). We set $u=K \omega$. Then $\operatorname{div} u=0$, and $u \cdot n(t, x)=0$ for any $t \geq 0$ and $x \in \partial \Omega$. The former calculation tells us that

$$
\operatorname{rot}\left(\frac{\partial u}{\partial t}-(u \cdot \nabla) u-\frac{1}{2} \Delta u\right)=0 .
$$

Hence there exists a function $p$ such that

$$
\frac{\partial u}{\partial t}-(u \cdot \nabla) u-\frac{1}{2} \Delta u=-\nabla p
$$

We have proved that $u$ solves (N.S.)'

We have now to characterize among the solutions $\omega$ of (1.8), the unique function such that $u=K \omega$ solves (N.S.), i.e.

$$
K \omega \cdot \tau(x)=0, \quad \text { for all } t \geq 0, \text { for all } x \in \partial \Omega,
$$

where $\tau(x)$ denotes the tangent vector at $x \in \partial \Omega$.

Lemma 1.2. Let $\omega$ be a solution of (1.8) and $u=K \omega$. The four following assertions are equivalent:

a) $u(t, x)=0$, for all $t \geq 0$, for all $x \in \partial \Omega$.

b) $\frac{\partial}{\partial n} \int_{\Omega} G\left(z, z^{\prime}\right) \omega\left(t, z^{\prime}\right) d z^{\prime}=0$, for all $t \geq 0$, for all $z \in \partial \Omega$.

c) $\int_{\Omega} h(z) \omega(t, z) d z=0$, for any bounded and harmonic function $h$ defined on $\Omega$.

$$
\begin{aligned}
\text { d) } \int_{\Omega} h(t, z) \omega(t, z) d z=0, \text { for any } h: \mathbb{R}_{+} \times \bar{\Omega} \longrightarrow \mathbb{R} \text { verifying } \\
\frac{1}{2} \Delta h \pm K \omega \cdot \nabla h=0 .
\end{aligned}
$$

Proof. 1) Recall that $u=K \omega$ verifies (N.S.)'. Moreover

$$
u \cdot \tau=\nabla^{\perp} \psi \cdot \tau= \pm \nabla \psi \cdot n= \pm \frac{\partial}{\partial n} \psi
$$


Since $\psi$ is given by (1.12), a) if and only if b) follows immediately.

2) Let $h: \mathbb{R}_{+} \times \bar{\Omega} \longrightarrow \mathbb{R}$, such that $\Delta h=\alpha K \omega \cdot \nabla h$ in $] 0, \infty[\times \Omega$, where $\alpha$ is a constant.

The Stokes formula and (1.11) tell us

$$
\begin{aligned}
\int_{\Omega} h(t, z) \omega(t, z) d z \\
\quad=\int_{\Omega} h(t, z) \Delta \psi(t, z) d z \\
\quad=\int_{\Omega} \Delta h(t, z) \psi(t, z) d z+\int_{\partial \Omega} h \frac{\partial \psi}{\partial n}-\int_{\partial \Omega} \psi \frac{\partial h}{\partial n} \\
\quad=\alpha \int_{\Omega} K \omega \cdot \nabla h(t, z) \psi(t, z) d z+\int_{\partial \Omega} h \frac{\partial \psi}{\partial n}-\int_{\partial \Omega} \psi \frac{\partial h}{\partial n} .
\end{aligned}
$$

We calculate the first integral by integrating by parts, we obtain,

$$
\begin{aligned}
\int_{\Omega}(K \omega & \nabla h)(t, z) \psi(t, z) d z \\
& =-\int_{\Omega}(\psi h \operatorname{div}(K \omega))(t, z) d z-\int_{\Omega}(h(K \omega \cdot \nabla \psi))(t, z) d z
\end{aligned}
$$

But $\psi=0$ on $\mathbb{R}_{+} \times \partial \Omega, \operatorname{div} K \omega=\operatorname{div} u=0$ and $K \omega \cdot \nabla \psi=\nabla^{\perp} \psi \cdot \nabla \psi=$ 0 , as a result

$$
\int_{\Omega} h(t, z) \omega(t, z) d z=\int_{\partial \Omega} h(t, \cdot) \frac{\partial \psi}{\partial n}
$$

It is now clear that b) if and only if c) if and only if d).

We will say that $u$ (or $\omega)$ is a solution of (N.S.) if,

$$
u=K \omega, \omega \text { solving the vorticity equation (1.8), }
$$

(1.16) and $u$ verifies one of the four conditions of Lemma 1.2. 


\section{The vorticity equation as a Kolmogorov equation.}

Let $u$ be the solution of the Navier Stokes system (N.S.), $\omega$ denotes the vorticity, $\omega=\operatorname{rot} u$.

The operator

$$
\mathcal{L}(f)=\frac{1}{2} \Delta f-u \cdot \nabla f
$$

is the generator of a diffusion process $D$. If $\Omega=\mathbb{R}^{2}$, it is classical to represent $\omega$ through $\omega_{0}$ and $D$, the crucial fact being that $\omega\left(t-s, D_{s}\right)$ is a martingale.

Here $\Omega$ is an open, bounded, simply connected open set. We suppose moreover that the boundary is smooth. Let us introduce the following reflected stochastic differential equation driven by a twodimensional Brownian motion $\left(B_{t}, t \geq 0\right)$, started at 0 , and defined on a probability space $\left(\Omega_{0},\left(\mathcal{F}_{t}\right)_{t \geq 0}, P\right)$

$$
\left\{\begin{array}{rlrl}
\widetilde{X}_{s}^{t, x}= & x+B_{s}-\int_{0}^{s} u\left(t-r, \widetilde{X}_{r}^{t, x}\right) d r & \\
-\int_{0}^{s} n\left(\widetilde{X}_{r}^{t, x}\right) d \widetilde{A}_{r}^{t, x}, & & 0 \leq s \leq t, \\
\int_{0}^{s} \mathbf{1}_{\left\{\tilde{X}_{r}^{t, x} \in \partial \Omega\right\}} d \widetilde{A}_{r}^{t, x}=\widetilde{A}_{s}^{t, x}, & \text { for all } s \in[0, t],
\end{array}\right.
$$

$u$ being a smooth function and $\partial \Omega$ being of class at less $C^{2}$, there exists a unique strong solution $\left(\widetilde{X}_{s}^{t, x} ; 0 \leq s \leq t\right)$ of $(2.1)$, taking its values in $\bar{\Omega}$, for any $t>0$ and $x \in \bar{\Omega}(c f . \overline{\mathrm{P}]}$ or $[\mathrm{SV}])$. Recall that $n(x)$ is the normalized outer normal vector at $x \in \partial \Omega$.

The solution of (2.1) is denoted $\tilde{X}$, because we will see in Section 3 that there exists a process $X$ such that $X$ and $\tilde{X}$ are dual processes.

The drift coefficient $-u$ corresponds to the mean velocity of $\widetilde{X}$; if $\Omega=\mathbb{R}^{2}$, it is easy to check that

$$
u\left(t-s, x_{0}\right)=-\lim _{h \rightarrow 0_{+}} E\left[\frac{\widetilde{X}_{s+h}^{t, x}-\tilde{X}_{s}^{t, x}}{h} \mid \mathcal{F}_{s}\right], \quad 0 \leq s \leq t .
$$

Recall that if $u$ is a solution of (N.S.)' (the weak form of (N.S.)), then $u \cdot n=0$ on $\mathbb{R}_{+} \times \partial \Omega$, and $u$ solves (N.S.) if $u \cdot \tau=0$ on $\mathbb{R}_{+} \times \partial \Omega$. If 
$\Omega=\mathbb{R} \times] 0,+\infty\left[(\Omega\right.$ is not bounded $)$, then $\partial \Omega=\left\{\left(x_{1}, x_{2}\right): x_{2}=0\right\}$. We choose $\rho_{0}\left(x_{1}, x_{2}\right)=x_{1}$. The analog of (2.2) would be

$$
(u \cdot \tau)\left(t-s, x_{0}\right)=\lim _{h \rightarrow 0} E\left[\frac{\rho_{0}\left(\widetilde{X}_{s+h}^{t, x}\right)-\rho_{0}\left(\widetilde{X}_{s}^{t, x}\right)}{h} \mid \mathcal{F}_{s}\right],
$$

where $0 \leq s \leq t, x \in \partial \Omega$. Our situation is more complicated. The function $\rho_{0}$ is replaced by the set $\mathcal{V}$ of velocity test functions. $\rho: \bar{\Omega} \longrightarrow$ $\mathbb{R}$ belongs to $\mathcal{V}$, if $\rho$ is of class $C^{\infty}$ and

$$
\begin{gathered}
\frac{\partial \rho}{\partial n}(z)=0, \quad \text { for all } z \in \partial \Omega, \\
\frac{\partial \rho}{\partial \tau} \neq 0 \text { almost sure on } \partial \Omega,
\end{gathered}
$$

We note that it is not possible to choose $\rho$ such that $(\partial \rho / \partial \tau)(z) \neq 0$ for all $z \in \partial \Omega$. If $(\partial \rho / \partial \tau)$ never vanishes on $\partial \Omega, \partial \rho / \partial \tau$ being continuous we suppose without loss of generality that $\partial \rho / \partial \tau>0$. Let $\gamma:[0,1] \longrightarrow \partial \Omega$ be a parametrization of $\partial \Omega$. Since $t \longrightarrow \rho(\gamma(t))$ is increasing, then $\rho(\gamma(0))<\rho(\gamma(1))$, this generates a contradiction with $\gamma(0)=\gamma(1)$.

Two objects play a crucial role in our approach. The first one is

$$
\varphi_{c}(s, x)=\frac{\frac{\partial \omega}{\partial n}}{\omega+c}(s, x), \quad s \geq 0, x \in \partial \Omega,
$$

where $c$ is a positive constant such that $\omega+c>0$.

The second one is the stochastic process

$$
\widetilde{Z}_{c}^{t, x}(s)=\left(\omega\left(t-s, \widetilde{X}_{s}^{t, x}\right)+c\right) \exp \left(\int_{0}^{s} \varphi_{c}\left(t-r, \widetilde{X}_{r}^{t, x}\right) d \widetilde{A}_{r}^{t, x}\right),
$$

where $0 \leq s \leq t$.

We are now ready to state the martingale property concerning $\omega$.

Proposition 2.1. Suppose $t>0, x \in \bar{\Omega}$ and $\omega$ is the vorticity solution of (1.8). where

1) a) $\left(\widetilde{Z}_{0}^{t, x}(s \wedge \widetilde{\xi}) ; 0 \leq s \leq t\right)$ is a continuous local martingale

$$
\widetilde{\xi}=\inf \left\{s \leq t: \widetilde{X}_{s}^{t, x} \in \partial \Omega \text { and } \omega\left(t-s ; \widetilde{X}_{s}^{t, x}\right)=0\right\} \wedge t
$$


(We assume the convention $\inf \phi=+\infty$ ).

b) If $c$ is large enough (i.e. $\left.c>C_{t, \Omega}\right),\left(\widetilde{Z}_{c}^{t, x}(s) ; 0 \leq s \leq t\right)$ is a square integrable continuous martingale, $C_{t, \Omega}$ being defined as

$$
C_{t, \Omega}=-\min \{\omega(s, x): 0 \leq s \leq t, x \in \Omega\} .
$$

2) For any positive $t, x$ in $\Omega$ and velocity test function $\rho$,

$$
\frac{1}{h} E\left[\rho\left(\widetilde{X}_{(\widetilde{T}+h) \wedge t}^{t, x}-\rho\left(\widetilde{X}_{\widetilde{T}}^{t, x}\right) \mid \mathcal{F}_{\widetilde{T}}\right] \underset{h \rightarrow 0}{\stackrel{\text { a.s. }}{\longrightarrow}} 0,\right.
$$

with,

$$
\widetilde{T}=\inf \left\{s \leq t: \tilde{X}_{s}^{t, x} \in \partial \Omega\right\} \wedge t
$$

Proof. 1) $t$ and $x$ being fixed, we denote for simplicity $\widetilde{X}=\widetilde{X}^{t, x}$ and $\widetilde{A}=\widetilde{A}^{t, x}$.

We apply the Itô formula

$$
\begin{aligned}
\omega\left(t-s, \widetilde{X}_{s}\right)= & \omega(t, x)+\int_{0}^{s} \nabla \omega\left(t-r, \widetilde{X}_{r}\right) d B_{r}-\int_{0}^{s} \frac{\partial \omega}{\partial n}\left(t-r, \widetilde{X}_{r}\right) d \widetilde{A}_{r} \\
& +\int_{0}^{s}\left(-\frac{\partial \omega}{\partial t}-u \cdot \nabla \omega+\frac{1}{2} \Delta \omega\right)\left(t-r, \widetilde{X}_{r}\right) d r
\end{aligned}
$$

where $0 \leq s \leq t$. $\omega$ solves (1.8), therefore,

$$
\begin{aligned}
\omega\left(t-s, \widetilde{X}_{s}\right)= & \omega(t, x)+\int_{0}^{s} \nabla \omega\left(t-r, \widetilde{X}_{r}\right) d B_{r} \\
& -\int_{0}^{s} \frac{\partial \omega}{\partial n}\left(t-r, \widetilde{X}_{r}\right) d \widetilde{A}_{r} .
\end{aligned}
$$

Let us introduce, for all integer $n \geq 1$

$$
\widetilde{\xi}_{n}=\inf \left\{s \leq t:\left|\omega\left(t-s, \widetilde{X}_{s}\right)\right| \leq \frac{1}{n} \text { and } \widetilde{X}_{s} \in \partial \Omega\right\} \wedge t
$$

$\left\{\widetilde{\xi}_{n}\right\}_{n \geq 1}$ is an increasing sequence of stopping times converging to $\widetilde{\xi}$. We set

$$
\widetilde{M}_{c}(s)=\exp \left(\int_{0}^{s} \varphi_{c}\left(t-r, \widetilde{X}_{r}\right) d \widetilde{A}_{r}\right)
$$


Using again the Itô formula we get,

$$
\begin{aligned}
& \omega\left(t-s \wedge \widetilde{\xi}_{n}, \widetilde{X}\left(s \wedge \widetilde{\xi}_{n}\right)\right) \widetilde{M}_{0}\left(s \wedge \widetilde{\xi}_{n}\right) \\
& =\omega(t, x)+\int_{0}^{s \wedge \widetilde{\xi}_{n}} \widetilde{M}_{0}(r) \nabla \omega\left(t-r, \widetilde{X}_{r}\right) d B_{r} \\
& \quad+\int_{0}^{s \wedge \widetilde{\xi}_{n}}\left(-\widetilde{M}_{0}(r) \frac{\partial \omega}{\partial n}\left(t-r, \widetilde{X}_{r}\right)+\widetilde{M}_{0}(r)\left(\omega \varphi_{0}\right)\left(t-r, \widetilde{X}_{r}\right)\right) d \widetilde{A}_{r} .
\end{aligned}
$$

But $\partial \omega / \partial n-\omega \varphi_{0}=0$, then

$$
\widetilde{Z}_{0}^{t, x}\left(s \wedge \widetilde{\xi}_{n}\right)=\omega(t, x)+\int_{0}^{s \wedge \widetilde{\xi}_{n}} \widetilde{M}_{0}(r) \nabla \omega\left(t-r, \widetilde{X}_{r}\right) d B_{r}
$$

Part 1) a) follows immediately.

We note that $(\omega+c)(s, y) \geq \alpha>0$ for any $(s, y) \in[0, t] \times \Omega, \omega+c$ solves (1.8) and

$$
\frac{\partial(\omega+c)}{\partial n}-(\omega+c) \varphi_{c}=0
$$

the former proof tells us that $\left(\widetilde{Z}_{c}^{t, x}(s) ; 0 \leq s \leq t\right)$ is a local martingale and

$$
\widetilde{Z}_{c}^{t, x}(s)=\omega(t, x)+c+\int_{0}^{s} \widetilde{M}_{c}(r) \nabla \omega\left(t-r, \widetilde{X}_{r}\right) d B_{r}
$$

We write

$$
\nabla \omega=(\omega+c) \frac{\nabla \omega}{\omega+c} .
$$

The function $\nabla \omega /(\omega+c)$ being bounded on $[0, t] \times \Omega$, making use of localization we have

$$
E\left[\left(\widetilde{Z}_{c}^{t, x}(s)\right)^{2}\right] \leq(\omega(t, x)+c)^{2}+K \int_{0}^{s} E\left[\left(\widetilde{Z}_{c}^{t, x}(r)\right)^{2}\right] d r .
$$

Gronwall lemma implies $E\left[\left(\widetilde{Z}_{c}^{t, x}(s)\right)^{2}\right] \leq(\omega(t, x)+c)^{2} e^{K s}, 0 \leq s \leq t$. This shows 1) b).

2) Let $\rho$ be a function of class $C^{\infty}$ verifying (2.4)-(2.6) (i.e. $\rho$ is a velocity test function). We apply the Itô formula to $\rho$

$$
\begin{aligned}
\rho\left(\widetilde{X}_{s}^{t, x}\right)= & \rho(x)+\int_{0}^{s} \nabla \rho\left(\widetilde{X}_{r}^{t, x}\right) d B_{r} \\
& -\int_{0}^{s}\left(u\left(t-r, \widetilde{X}_{r}^{t, x}\right) \cdot \nabla \rho\left(\widetilde{X}_{r}^{t, x}\right)-\frac{1}{2} \Delta \rho\left(\widetilde{X}_{r}^{t, x}\right)\right) d r .
\end{aligned}
$$


Consequently (for simplicity we write $T$ for $\widetilde{T}$ ).

$$
\begin{aligned}
& \rho\left(\tilde{X}_{(T+h) \wedge t}^{t, x}\right)-\rho\left(\tilde{X}_{T}^{t, x}\right) \\
& =\int_{T}^{(T+h) \wedge t} \nabla \rho\left(\widetilde{X}_{r}^{t, x}\right) d B_{r} \\
& \quad-\int_{T}^{(T+h) \wedge t}\left(u\left(t-r, \widetilde{X}_{r}^{t, x}\right) \cdot \nabla \rho\left(\widetilde{X}_{r}^{t, x}\right)-\frac{1}{2} \Delta \rho\left(\widetilde{X}_{r}^{t, x}\right)\right) d r, \\
& \quad \frac{1}{h} E\left[\rho\left(\widetilde{X}_{(T+h) \wedge t}^{t, x}\right)-\rho\left(\widetilde{X}_{T}^{t, x}\right) \mid \mathcal{F}_{T}\right] \\
& =-\frac{1}{h} E\left[\int _ { T } ^ { ( T + h ) \wedge t } \left(u\left(t-r, \widetilde{X}_{r}^{t, x}\right) \cdot \nabla \rho\left(\widetilde{X}_{r}^{t, x}\right)\right.\right. \\
& \left.\left.\quad-\frac{1}{2} \Delta \rho\left(\widetilde{X}_{r}^{t, x}\right) d r\right) \mid \mathcal{F}_{T}\right] .
\end{aligned}
$$

On $\{T=t\} \in \mathcal{F}_{T}$, the integral is equal to 0 , therefore the limit is 0 . Suppose $\{T<t\}$. Recall that $\Delta \rho=0$ in a neighbourhood of $\partial \Omega$, $u$ and $\nabla \rho$ are continuous functions, then the almost sure limit of the right-hand side of $(2.18)$ is $-u\left(t-T, \widetilde{X}_{T}^{t, x}\right) \cdot \nabla \rho\left(\widetilde{X}_{T}^{t, x}\right)$. But on $\{T<t\}$, $\widetilde{X}_{T}^{t, x} \in \partial \Omega$ then $u\left(t-T, \widetilde{X}_{T}^{t, x}\right)=0$.

Applying the stopping theorem we get:

Corollary 2.2. Recall that $\widetilde{\xi}_{n}$ is the stopping time defined by (2.14). Then

$$
\begin{aligned}
& \omega(t, x)=E\left[\omega\left(t-s \wedge \widetilde{\xi}_{n}, \widetilde{X}^{t, x}\left(s \wedge \widetilde{\xi}_{n}\right)\right)\right. \\
& \left.\cdot \exp \left(\int_{0}^{s \wedge \widetilde{\xi}_{n}} \varphi_{0}\left(t-r, \widetilde{X}_{r}^{t, x}\right) d \widetilde{A}_{r}^{t, x}\right)\right], \\
& \begin{aligned}
\omega(t, x)+c=E\left[\left(\omega\left(t-s, \widetilde{X}_{s}^{t, x}\right)+c\right)\right. \\
\left.\cdot \exp \left(\int_{0}^{s} \varphi_{c}\left(t-r, \widetilde{X}_{r}^{t, x}\right) d \widetilde{A}_{r}^{t, x}\right)\right],
\end{aligned}
\end{aligned}
$$

$c$ being larger than $C_{t, \Omega}$. 
We would like to define a self-contained nonlinear stochastic system -we call it the Stochastic Navier Stokes system (S.N.S.)- equivalent to the Navier Stokes system. Proposition 2.1, tells us that $\widetilde{X}$ is a good candidate concerning the stochastic part. It remains to express the drift term $K \omega$ through the underlying process $\widetilde{X}$.

Let us detail the (S.N.S.) system and its three conditions (S.N.S.1), (S.N.S.2) and (S.N.S.3). The unknown parameters are $\omega,\left\{\left(\widetilde{X}_{s}^{t, x} ; 0 \leq\right.\right.$ $\left.s \leq t),\left(\widetilde{A}_{s}^{t, x} ; 0 \leq s \leq t\right), t \geq 0, x \in \bar{\Omega}\right\}$.

(S.N.S.1) For any positive $t$ and $x$ in $\bar{\Omega}$, consider the following reflected stochastic differential equation

$$
\begin{aligned}
\widetilde{X}_{s}^{t, x}= & x+B_{s}-\int_{0}^{s} K \omega\left(t-r, \widetilde{X}_{r}^{t, x}\right) d r \\
& -\int_{0}^{s} n\left(\widetilde{X}_{r}^{t, x}\right) d \widetilde{A}_{r}^{t, x}, \quad 0 \leq s \leq t,
\end{aligned}
$$

$$
\int_{0}^{s} 1_{\left\{\widetilde{X}_{r}^{t, x} \in \partial \Omega\right\}} d \widetilde{A}_{r}^{t, x}=\widetilde{A}_{s}^{t, x}, \quad \text { for all } s \in[0, t] .
$$

$\left(\widetilde{A}_{s}^{t, x} ; 0 \leq s \leq t\right)$ is the local time of $\widetilde{X}^{t, x}$ on the boundary.

Recall that $K \omega$ is the function defined by (1.2).

(S.N.S.2) The process $\left(\omega\left(t-s \wedge \widetilde{T}, \widetilde{X}^{t, x}(s \wedge \widetilde{T})\right)\right)$ is a martingale, for any $t>0$ and $x \in \bar{\Omega}$, where $\widetilde{T}$ is the first hitting time of the boundary

$$
\widetilde{T}=\inf \left\{s \leq t: \widetilde{X}^{t, x}(s) \in \partial \Omega\right\}
$$

(S.N.S.3) For any $t>0$ and velocity test function $\rho$

$$
\frac{1}{h} E\left[\rho\left(\widetilde{X}_{\widetilde{T}+h}^{t, x}\right)-\rho\left(\widetilde{X}_{\widetilde{T}}^{t, x}\right) \mid \mathcal{F}_{\widetilde{T}} \underset{h \rightarrow 0}{\stackrel{\text { a.s. }}{\longrightarrow}} 0,\right.
$$

for any $x$ in $\Omega$.

We just now state the converse of Proposition 2.1.

Proposition 2.3. Suppose that (S.N.S.) has a unique solution

$$
\left(\omega,\left\{\widetilde{X}_{s}^{t, x}, \widetilde{A}_{s}^{t, x} ; 0 \leq s \leq t\right\}, t \geq 0, x \in \bar{\Omega}\right),
$$


where $\omega$ is smooth. Then $u=K \omega$ is a solution of (N.S.) equation (i.e. verifies (1.15) and (1.16)).

Proof. 1) $\omega$ is bounded on $[0, t] \times \bar{\Omega}$, therefore $\left(\omega\left(t-s \wedge \widetilde{T}, \widetilde{X}^{t, x}(s \wedge\right.\right.$ $\widetilde{T}) ; 0 \leq s \leq t)$ ) is a bounded martingale. By the stopping theorem,

$$
\omega(t, x)=E\left[\omega\left(t-s \wedge \widetilde{T}, \widetilde{X}^{t, x}(s \wedge \widetilde{T})\right)\right], \quad 0 \leq s \leq t .
$$

The infinitesimal generator of $\left(\left(t-s ; \widetilde{X}_{s}^{t, x}\right) ; 0 \leq s \leq t\right)$ is $\mathcal{L}$, with

$$
\mathcal{L} f(s, x)=\left(-\frac{\partial f}{\partial s}+\frac{1}{2} \Delta f-u \cdot \nabla f\right)(t-s, x) .
$$

(2.23) implies that $\omega$ is the solution to the Dirichlet problem in $[0, t] \times \bar{\Omega}$ associated with $\mathcal{L}$. Consequently $\omega$ solves the vorticity equation (1.8).

2) Let $\rho$ be a velocity test function (recall that $\rho$ is of class $C^{\infty}$ and solves (2.4)-(2.6)). We are allowed to use relation (2.18)

$$
\begin{aligned}
\frac{1}{h} E\left[\rho\left(\widetilde{X}_{\widetilde{T}+h}^{t, x}\right)\right. & \left.-\rho\left(\widetilde{X}_{\widetilde{T}}^{t, x}\right) \mid \mathcal{F}_{\widetilde{T}}\right] \\
& =-\frac{1}{h} E\left[\int_{\widetilde{T}}^{\widetilde{T}+h} K \omega\left(t-r, \widetilde{X}_{r}^{t, x}\right) \cdot \nabla \rho\left(\widetilde{X}_{r}^{t, x}\right) d r \mid \mathcal{F}_{\widetilde{T}}\right],
\end{aligned}
$$

since for $h$ small enough $\Delta \rho\left(\widetilde{X}_{r}^{t, x}\right)=0, \widetilde{T} \leq r \leq \widetilde{T}+h$.

We take the limit $h \rightarrow 0_{+}$, the functions $K \omega$ and $\nabla \rho$ being bounded, we have

$$
K \omega\left(t-\widetilde{T}, \widetilde{X}_{\widetilde{T}}^{t, x}\right) \cdot \nabla \rho\left(\widetilde{X}_{\widetilde{T}}^{t, x}\right)=0, \quad \text { almost sure } .
$$

From part 1) of Lemma 1.1, we know that $(K \omega) \cdot n=0$ on $[0, t] \times \partial \Omega$. Consequently (2.24) is equivalent to

$$
\frac{\partial K \omega}{\partial n}\left(t-\widetilde{T}, \widetilde{X}_{\widetilde{T}}^{t, x}\right) \frac{\partial \rho}{\partial n}\left(\widetilde{X}_{\widetilde{T}}^{t, x}\right)=0, \quad \text { almost sure } .
$$

Since $x$ belongs to $\Omega$, conditionnally to $\{\widetilde{T}<t\}$, the distribution of $\left(\widetilde{T}, \widetilde{X}_{\widetilde{T}}^{t, x}\right)$ is absolutely continuous with respect to $\left(\mathbf{1}_{[0, t]}(u) d u\right) \otimes \widetilde{\lambda}(d v)$, where $\widetilde{\lambda}$ denotes the Lebesgue measure on $\partial \Omega$. Assumption (2.5) implies

$$
\frac{\partial K \omega}{\partial n}(t-s, y)=0, \quad \text { for almost every }(s, y) \in[0, t] \times \partial \Omega .
$$


$\partial K \omega / \partial n$ being a continuous function, the former condition is equivalent to $(\partial K \omega / \partial n)(s, y)=0$ for any $s \in[0, t]$ and $y \in \partial \Omega$. $u$ is a solution of the (N.S.) equation because $K \omega$ vanishes on $\mathbb{R}_{+} \times \partial \Omega$.

REMARKS 2.4. 1) (S.N.S.2) reveals the nonlinearity of $\widetilde{X}$ inside $\Omega$. Indeed, (2.21) shows that $\widetilde{X}$ depends on $\omega$; and the martingale property (S.N.S.2) involving $\omega$ depends on $\widetilde{X}$.

2) (S.N.S.3) implies that the tangential component $u \cdot \tau$ of $u=K \omega$ is equal to 0 on the boundary of $\Omega$.

3) A priori, 1) a) of Proposition 2.1 seems a stronger condition than (S.N.S.2). We claim that these two conditions are equivalent.

We remark that $\widetilde{Z}_{0}^{t, x}(t \wedge \widetilde{T})=\omega\left(t-s \wedge \widetilde{T}, \widetilde{X}_{s \wedge \widetilde{T}}^{t, x}\right)$, where $\widetilde{T}$ is the first hitting time of $\partial \Omega$, and $\widetilde{T} \leq \widetilde{\xi}$ and $\widetilde{\xi}$ is the stopping time defined by (2.9). Therefore if $\omega$ solves the vorticity equation (1.8), $\left(\widetilde{Z}_{0}^{t, x}(s \wedge \widetilde{\xi}) ; 0 \leq s \leq t\right)$ is a local martingale, then (S.N.S.2) holds.

Let us analyze the converse. Suppose that the (S.N.S.) system has a solution. Hence $\omega$ solves (1.8). Applying the Itô formula we have,

$$
\begin{aligned}
\omega\left(t-s, \widetilde{X}_{s}^{t, x}\right)= & \omega(t, x)+\int_{0}^{s} \nabla \omega\left(t-r, \widetilde{X}_{r}^{t, x}\right) d B_{r} \\
& -\int_{0}^{s} \frac{\partial \omega}{\partial n}\left(t-r, \widetilde{X}_{r}^{t, x}\right) d \widetilde{A}_{r}^{t, x} .
\end{aligned}
$$

Recall that

$$
\varphi_{0}=\frac{1}{\omega} \frac{\partial \omega}{\partial n}
$$

using again the Itô formula we obtain,

$$
\begin{aligned}
\widetilde{Z}_{0}^{t, x}(s)=\omega\left(t-s, \widetilde{X}_{s}^{t, s}\right) \exp \int_{0}^{s} \varphi_{0}\left(t-r, \widetilde{X}_{r}^{t, x}\right) d \widetilde{A}_{r}^{t, x} \\
=\omega(t, x)+\int_{0}^{s} \nabla \omega\left(t-u, \widetilde{X}_{u}^{t, x}\right) \\
\quad \cdot \exp \left(\int_{0}^{u} \varphi_{0}\left(t-r, \widetilde{X}_{r}^{t, x}\right) d \widetilde{A}_{r}^{t, x}\right) d B_{u},
\end{aligned}
$$

$s$ belonging to the stochastic interval $[0, \widetilde{\xi}]$.

This proves that $\left(\widetilde{Z}_{0}^{t, x}(s \wedge \widetilde{\xi}) ; 0 \leq s \leq \widetilde{\xi}\right)$ is a local martingale (i.e. 1) a) of Proposition 2.1 holds). 
We analyze the integrability of $\widetilde{A}^{t, x}$. This plays an important role in Lemma 4.2.

Proposition 2.5. For any $\theta>0, t>0$ and $x \in \bar{\Omega}$,

$$
E\left[\exp \left(\theta \widetilde{A}_{t}^{t, x}\right)\right]<\infty .
$$

Moreover, for any $k$,

$$
\lim _{u \rightarrow 0} \sup _{\substack{0 \leq t \leq k \\ x \in \bar{\Omega}}} E\left[\widetilde{A}_{u}^{t, x}\right]=0 .
$$

REMARKS. 1) In dimension 1, for the reflected Brownian motion, recall that the local time at $0, L_{t}^{0}$, has exponential moments, since $L_{t}^{0} \stackrel{(\mathrm{d})}{=}$ $\sqrt{t}|N|$, where $N$ is a centered, unit variance, Gaussian random variable.

2) A similar estimation can be found in [S.V.].

Proof of Proposition 2.5. 1) Let $\lambda>0$ be fixed.

We choose $\gamma: \bar{\Omega} \longrightarrow \mathbb{R}$, a function of class $C^{2}$ such that,

$$
\begin{aligned}
& \gamma(x) \geq 1, \quad \text { for all } x \in \bar{\Omega} . \\
& \text { i) } \frac{\partial \gamma}{\partial n}(x)=2 \lambda \\
& \text { ii) } \gamma(x)=2, \text { for any } x \in \partial \Omega \text {. }
\end{aligned}
$$

A straightforward calculation based on the Itô formula and (2.28) shows that $\left(U_{s} ; 0 \leq s \leq t\right)$ is a bounded martingale, where $\widetilde{X}_{s}=\widetilde{X}_{s}^{t, x}$, $\widetilde{A}_{s}=\widetilde{A}_{s}^{t, x}$ and

$$
\begin{gathered}
U_{s}=\gamma\left(\widetilde{X}_{s}\right) \exp \left(\lambda \widetilde{A}_{s}\right)-\int_{0}^{s} H(r) \exp \left(\lambda \widetilde{A}_{r}\right) d r \\
H(r)=\frac{1}{2} \Delta \gamma\left(\widetilde{X}_{r}\right)-u\left(t-r, \widetilde{X}_{r}\right) \nabla \gamma\left(\widetilde{X}_{r}\right) .
\end{gathered}
$$

$\gamma$ being of class $C^{2}, H(r)$ is a bounded process, then there exists a positive constant $k$ such that $|H(r)| \leq k$ for any $r$ in $[0, t]$. (2.27) implies that

$$
H(r) \leq|H(r)| \leq k \gamma\left(\widetilde{X}_{r}\right), \quad \text { for all } r \in[0, t] .
$$


Let $\left\{T_{n}\right\}_{n \geq 1}$ be a increasing sequence of stopping times, converging to $t$ such that $\widetilde{A}_{t \wedge T_{n}} \leq n$.

We replace $s$ by $s \wedge T_{n}$ in (2.29) and we take the expectation, we easily obtain the following inequality

$$
\begin{aligned}
\gamma(x) & =E\left[U_{s \wedge T_{n}}\right] \\
& \geq E\left[\gamma\left(\widetilde{X}_{s \wedge T_{n}}\right) \exp \left(\lambda \widetilde{A}_{s \wedge T_{n}}\right)\right]-k E\left[\int_{0}^{s \wedge T n} \gamma\left(\widetilde{X}_{r}\right) \exp \left(\lambda \widetilde{A}_{r}\right) d r\right] .
\end{aligned}
$$

We set $\alpha_{n}(s)=E\left[\gamma\left(\widetilde{X}_{s \wedge T_{n}}\right) \exp \left(\lambda \widetilde{A}_{s \wedge T_{n}}\right)\right]$. The former inequality is equivalent to

$$
\alpha_{n}(s) \leq \gamma(x)+k \int_{0}^{s} \alpha_{n}(u) d u, \quad \text { for all } s \in[0, t] .
$$

Using the Gronwall lemma and (2.27) we conclude that

$$
E\left[\exp \lambda \widetilde{A}_{s \wedge T_{n}}\right] \leq \gamma(x) e^{k s} .
$$

We take the limit $n$ going to infinity,

$$
E\left[\exp \lambda \widetilde{A}_{s}\right] \leq \gamma(x) e^{k s} .
$$

2) As for $(2.26)$, we take $\gamma_{0}: \bar{\Omega} \longrightarrow \mathbb{R}$ of class $C^{2}$, such that

$$
\frac{\partial \gamma_{0}}{\partial n}(x)=1, \quad \text { for all } x \in \partial \Omega .
$$

We apply the Itô formula and we take the expectation

$$
\begin{gathered}
E\left[\widetilde{A}_{s}^{t, x}\right]=E\left[\gamma_{0}(x)-\gamma_{0}\left(\widetilde{X}_{s}^{t, x}\right)\right]+\int_{0}^{s} h(r) d r, \\
h(r)=E\left[\frac{1}{2} \Delta \gamma_{0}\left(\widetilde{X}_{r}^{t, x}\right)-u\left(t-r, \widetilde{X}_{r}^{t, x}\right) \nabla \gamma_{0}\left(\widetilde{X}_{r}^{t, x}\right)\right] .
\end{gathered}
$$

Since $\gamma_{0}$ is of class $C^{2}, h$ is bounded, moreover the continuity of $(t, x, s)$ $\longrightarrow \widetilde{X}_{s}^{t, x}$ implies that $(2.26)$ is verified.

Before ending this section we prove some properties concerning the distribution of $\widetilde{X}_{s}^{t, x}$ (respectively $\int_{0}^{t} H\left(r, \widetilde{X}_{r}^{t}\right) d \widetilde{A}_{r}^{t}$ ) when $\widetilde{X}_{0}^{t}$ is uniformly distributed on $\bar{\Omega}$. 
Notations. 1) $\lambda$ is the normalized Lebesgue measure on $\bar{\Omega}: \lambda$ is proportional to the Lebesgue measure on $\bar{\Omega}$ and $\lambda(\bar{\Omega})=1$.

2) Let $h: \bar{\Omega} \longrightarrow \mathbb{R}$ and $F: \mathcal{C}([0, t]) \longrightarrow \mathbb{R}$, we set

$$
\begin{aligned}
& E_{h \cdot \lambda}\left[F\left(\tilde{X}_{s}^{t} ; 0 \leq s \leq t\right)\right] \\
& \quad=\int_{\Omega} E\left[F\left(\widetilde{X}_{s}^{t, x} ; 0 \leq s \leq t\right)\right] h(x) \lambda(d x) .
\end{aligned}
$$

(2.30) is meaningfull if for instance $h$ and $F$ are positive.

Proposition 2.6. 1) Suppose $f: \bar{\Omega} \longrightarrow \mathbb{R}$ and $F:[0, t] \times \partial \Omega \longrightarrow \mathbb{R}$, $H:[0, t] \times \partial \Omega \longrightarrow \mathbb{R}$ are bounded Borel functions. Then, for any $s$ in $[0, t]$

$$
\begin{gathered}
E_{\lambda}\left[f\left(\tilde{X}_{s}^{t}\right)\right]=\int_{\Omega} f(x)(d x) \\
E_{\lambda}\left[\int_{0}^{s} H\left(r, \widetilde{X}_{r}^{t}\right) d \widetilde{A}_{r}^{t}\right]=\frac{1}{2} \int_{0}^{s}\left(\int_{\partial \Omega} H(r, x) d x\right) d r .
\end{gathered}
$$

Proof of Proposition 2.6. 1) The first identity is classical. Let $\widetilde{L}$ be the infinitesimal generator of $\widetilde{X}$ and $L$

$$
L(f)=\frac{1}{2} \Delta f+u \cdot \nabla f .
$$

$L$ and $\widetilde{L}$ are symmetric with respect to the probability measure $\lambda$ (see Section 3), therefore $\lambda$ is the invariant probability measure of $\widetilde{X}$.

2) We analyze (2.32). Let $g:[0, t] \times \bar{\Omega} \longrightarrow \mathbb{R}$ be of class $C^{2}$. We apply the Itô formula and we take the expectation

$$
\begin{aligned}
& E_{\lambda}\left[g\left(s, \widetilde{X}_{s}^{t}\right)\right] \\
& =\int_{\Omega} g(0, x) d \lambda(x)-E_{\lambda}\left[\int_{0}^{s} \frac{\partial g}{\partial n}\left(r, \widetilde{X}_{r}^{t}\right) d \widetilde{A}_{r}^{t}\right] \\
& \quad+E_{\lambda}\left[\int_{0}^{s}\left(\frac{1}{2} \Delta g(r, \cdot)-u(t-r, \cdot) \nabla g(r, \cdot)+\frac{\partial g}{\partial t}(r, \cdot)\right)\left(\widetilde{X}_{r}^{t}\right) d r\right] .
\end{aligned}
$$

We use the former identity (2.31)

$$
\left.E_{\lambda}\left[\int_{0}^{s} \frac{\partial g}{\partial n}\left(r, \widetilde{X}_{r}^{t}\right) d \widetilde{A}_{r}^{t}\right)\right]=A_{1}+A_{2}
$$


where

$$
\begin{gathered}
A_{1}=\int_{\Omega} g(0, x) d \lambda(x)-\int_{\Omega} g(s, x) d \lambda(x)+\int_{0}^{s}\left(\int_{\Omega} \frac{\partial g}{\partial t}(r, x) \lambda(d x)\right) d r \\
A_{2}=\int_{0}^{s}\left(\int_{\Omega}\left(\frac{1}{2} \Delta g(r, x)-u(t-r, x) \nabla g(t, x)\right) \lambda(d x)\right) d r .
\end{gathered}
$$

It is obvious that $A_{1}=0$.

We transform $A_{2}$ through Stokes formula, and an integration by parts,

$$
\begin{gathered}
\int_{\Omega} \Delta g(r, x) \lambda(d x)=\int_{\partial \Omega} \frac{\partial g}{\partial n}(r, x) d x \\
\int_{\Omega} u(t-r, x) \nabla g(r, x) \lambda(d x)=-\int_{\Omega} g(r, x) \operatorname{div} u(t-r, x) \lambda(d x)=0 .
\end{gathered}
$$

(Recall that $\operatorname{div} u=0$ ). Therefore

$$
E_{\lambda}\left[\int_{0}^{s} \frac{\partial g}{\partial n}\left(r, \widetilde{X}_{r}^{t}\right) d \widetilde{A}_{r}^{t}\right]=\frac{1}{2} \int_{0}^{s}\left(\int_{\partial \Omega} \frac{\partial g}{\partial n}(r, x) d x\right) d r .
$$

For any $g$, of class $C^{2}$. (2.32) is a direct consequence of the monotone class theorem.

\section{Fokker-Planck interpretation of the vorticity equation.}

Let $\omega$ be the vorticity associated with $u, u$ being the velocity solving the Navier Stokes system. Recall that $\omega$ solves (1.8).

We know that if $D$ is a diffusion process with drift term $b$, and coefficient of diffusion identically equal to 1 , the density $\varphi$ of $D$ verifies the Fokker-Planck equation

$$
\frac{\partial \varphi}{\partial t}=\frac{1}{2} \Delta \varphi-b \nabla \varphi-(\operatorname{div} b) \varphi, \quad t>0, x \in \Omega .
$$

Since $\operatorname{div} u=0$, if we choose $u=b$, we note that the vorticity equation can be written as a Fokker-Planck equation.

If $\Omega$ is equal to the whole space $\mathbb{R}^{2}, \omega$ is the "density" of $D, D$ starting with initial distribution $\omega_{0}(x) d x$

$$
\int_{\mathbb{R}^{2}} h(x) \omega(t, x) d x=\int_{\mathbb{R}^{2}} \omega_{0}(x) E\left[h\left(D_{t}^{x}\right)\right] d x,
$$


where $D^{x}$ solves,

$$
D_{t}^{x}=x+B_{t}+\int_{0}^{t} u\left(s, D_{s}^{x}\right) d s .
$$

$B$ being a two-dimensional Brownian motion, $B_{0}=0$.

In our context we guess that the underlying process associated with $\omega$ (or $u$ ) has to stay in $\bar{\Omega}$. It is a well-known problem solved by adding a local time process in the right-hand side of (3.3).

More precisely let $\mathcal{X}=\left(\left(X_{s}^{t, x} ; s \geq 0\right), x \in \bar{\Omega}, t \geq 0\right)$ be the family of diffusions, with normal reflection

$$
\left\{\begin{array}{l}
X_{s}^{t, x}=x+B_{s}+\int_{0}^{s} u\left(t+r, X_{r}^{t, x}\right) d r-\int_{0}^{s} n\left(X_{r}^{t, x}\right) d A_{r}^{t, x} \\
A_{s}^{t, x}=\int_{0}^{s} \mathbf{1}_{\left\{X_{r}^{t, x} \in \partial \Omega\right\}} d A_{r}^{t, x},
\end{array}\right.
$$

$n(y)$ is the normalized outer normal vector at $y \in \partial \Omega,\left(A_{s}^{t, x} ; s \geq 0\right)$ is the local time process corresponding to a normal reflection at the boundary. We know that $X_{s}^{t, x}$ belongs to $\bar{\Omega}$ for any $(s, t, x)$ in $\mathbb{R}_{+}^{2} \times \bar{\Omega}$.

The aim of this section is double. We prove that $\mathcal{X}$ and $\widetilde{\mathcal{X}}$ are in duality, $\widetilde{\mathcal{X}}=\left(\left(\widetilde{X}_{s}^{t, x} ; 0 \leq s \leq t\right) ;(t, x) \in \mathbb{R}_{+} \times \bar{\Omega}\right)$ being the family of stochastic processes introduced in Section 2, and especially in (2.1). We also come back to the interpretation of $\omega$ as a "density" function.

We keep the same notation we have introduced in (2.30)

$$
\begin{gathered}
E_{h \cdot \lambda}\left[F\left(\widetilde{X}_{s}^{t} ; 0 \leq s \leq t\right)\right] \\
\quad=\int_{\Omega} E\left[F\left(\widetilde{X}_{s}^{t, x} ; 0 \leq s \leq t\right)\right] h(x) \lambda(d x), \\
E_{h \cdot \lambda}\left[F\left(X_{s}^{t} ; s \geq 0\right)\right]=\int_{\Omega} E\left[F\left(X_{s}^{t, x} ; s \geq 0\right)\right] h(x) \lambda(d x),
\end{gathered}
$$

where $h: \bar{\Omega} \longrightarrow \mathbb{R}$ and $F: \mathcal{C}\left(\mathbb{R}_{+}\right) \longrightarrow \mathbb{R}$ are Borel functions. $\lambda$ denotes the normalized Lebesgue measure on $\Omega$.

We begin with the duality between $\mathcal{X}$ and $\widetilde{\mathcal{X}}$.

Proposition 3.1. i) Suppose $t>0$ and $F: \mathcal{C}([0, t]) \rightarrow \mathbb{R}$ be a Borel bounded function, then

$$
E_{\lambda}\left[F\left(X_{s}^{0} ; 0 \leq s \leq t\right)\right]=E_{\lambda}\left[F\left(\tilde{X}_{t-s}^{t} ; 0 \leq s \leq t\right)\right]
$$


ii) In particular if $0=s_{0}<s_{1}<\cdots<s_{n} \leq t$ and $f_{0}, \ldots, f_{n}: \bar{\Omega} \longrightarrow \mathbb{R}$ are bounded Borel functions we have

$$
\begin{aligned}
E_{\lambda}\left[f_{0}\left(X_{s_{0}}^{0}\right) f_{1}\left(X_{s_{1}}^{0}\right)\right. & \left.\cdots f_{n}\left(X_{s_{n}}^{0}\right)\right] \\
& =E_{\lambda}\left[f_{0}\left(\widetilde{X}_{t-s_{0}}^{t}\right) f_{1}\left(\widetilde{X}_{t-s_{1}}^{t}\right) \cdots f_{n}\left(\widetilde{X}_{t-s_{n}}^{t}\right)\right]
\end{aligned}
$$

Proof. The result is well-known if $X$ or $\tilde{X}$ are homogeneous Markov processes. Here they are not, therefore we briefly indicate the main steps of the proof. The monotone class theorem implies that it is sufficient to verify (3.8). Using the Markov property and induction we can reduce to $n=1$. We set

$$
\begin{aligned}
& \delta=E_{\lambda}\left[f_{0}\left(X_{s_{0}}^{0}\right) f_{1}\left(X_{s_{1}}^{0}\right)\right] \\
& \widetilde{\delta}=E_{\lambda}\left[f_{0}\left(\widetilde{X}_{t-s_{0}}^{t}\right) f_{1}\left(\widetilde{X}_{t-s_{1}}^{t}\right)\right], \\
& 0=s_{0}<s_{1} \leq t
\end{aligned}
$$

Let $\left(\wedge_{r, s}\right)_{0 \leq r<s}$ (respectively $\left.\left(\widetilde{\wedge}_{r, s}^{t}\right)_{0<r<s \leq t}\right)$ be the non homogeneous semigroup associated with $X$ (respectively $\widetilde{X}^{t}$ ).

We denote by $L_{r}$ and $\widetilde{L}_{r}^{t}$ the infinitesimal generators of $\wedge$ and $\widetilde{\wedge}$

$$
\begin{gathered}
L_{r} f(x)=\frac{1}{2} \Delta f(x)+u(r, x) \nabla f(x), \\
\widetilde{L}_{r}^{t} f(x)=\frac{1}{2} \Delta f(x)-u(t-r, x) \nabla f(x),
\end{gathered}
$$

for any $f$ of class $C^{2}$ in $\bar{\Omega}$, and verifying

$$
\frac{\partial f}{\partial n}=0, \quad \text { on } \partial \Omega
$$

1) We claim that

$$
\left\langle g, L_{r} f\right\rangle_{\lambda}=\left\langle f, \widetilde{L}_{t-r}^{t} g\right\rangle_{\lambda}, \quad 0<r<t
$$

where $f$ and $g$ are of class $C^{2}$,

$$
\frac{\partial f}{\partial n}=\frac{\partial g}{\partial n}=0
$$


and

$$
\langle f, g\rangle_{\lambda}=\int_{\Omega} f(x) g(x) \lambda(d x)
$$

We integrate by part, making use of Stokes formula

$$
\begin{aligned}
\int_{\Omega} g(x) L_{r} f(x) d x= & \frac{1}{2}\left(\int_{\Omega} f(x) \Delta g(x) d x+\int_{\partial \Omega} g \frac{\partial f}{\partial n}-\int_{\partial \Omega} f \frac{\partial g}{\partial n}\right) \\
& -\int_{\Omega} g(x) f(x) \operatorname{div} u(r, x) d x \\
& -\int_{\Omega} f(x)(u(r, x) \nabla g(x)) d x+\int_{\partial \Omega} u g f .
\end{aligned}
$$

Since $\partial f / \partial n, \partial g / \partial n, u_{\mid \partial \Omega}$ and $\operatorname{div} u$ cancel, we obtain (3.9).

2) In this second step, we prove

$$
\left\langle f, \widetilde{\wedge}_{t-s, t-r}^{t} g\right\rangle_{\lambda}=\left\langle\wedge_{r, s} f, g\right\rangle_{\lambda}, \quad 0 \leq r \leq s \leq t,
$$

for any $f$ and $g$ of class $C^{2}, \partial f / \partial n=\partial g / \partial n=0$.

We set

$$
\alpha(h)=\left\langle\wedge_{r, h} f, \widetilde{\wedge}_{t-s, t-h}^{t} g\right\rangle_{\lambda}, \quad h \in[r, s] .
$$

We take the derivative of $\alpha$

$$
\alpha^{\prime}(h)=\left\langle L_{h} \wedge_{r, h} f, \widetilde{\wedge}_{t-s, t-h}^{t} g\right\rangle_{\lambda}-\left\langle\wedge_{r, h} f, \widetilde{L}_{t-h} \widetilde{\wedge}_{t-s, t-h}^{t} g\right\rangle_{\lambda},
$$

because

$$
\frac{\partial}{\partial h} \wedge_{r, h} f=L_{h} \wedge_{r, h} f \quad \text { and } \quad \frac{\partial}{\partial h} \widetilde{\wedge}_{t-s, t-h}^{t}=-\widetilde{L}_{t-h} \widetilde{\wedge}_{t-s, t-h}^{t} .
$$

(3.9) implies that $\alpha^{\prime}(h)=0$, for all $h \in[r, s]$. Hence $\alpha$ is constant

$$
\alpha(r)=\left\langle f, \widetilde{\wedge}_{t-s, t-r}^{t} g\right\rangle_{\lambda}=\alpha(s)=\left\langle\wedge_{r, s} f, g\right\rangle_{\lambda} .
$$

3) We come back to $\delta$ and $\widetilde{\delta}$. We have

$$
\begin{gathered}
\delta=\left\langle f_{0}, \wedge_{0, s_{1}} f_{1}\right\rangle_{\lambda}, \\
\widetilde{\delta}=E_{\lambda}\left[f_{1}\left(\widetilde{X}_{t-s_{1}}^{t}\right) \widetilde{\wedge}_{t-s_{1}, t}^{t} f_{0}\left(\widetilde{X}_{t-s_{1}}^{t}\right)\right]=\left\langle 1, \widetilde{\wedge}_{0, t-s_{1}}^{t}\left(f_{1}\left(\widetilde{\wedge}_{t-s_{1}, t}^{t} f_{0}\right)\right)\right\rangle_{\lambda} .
\end{gathered}
$$


We apply twice (3.11),

$$
\widetilde{\delta}=\left\langle 1, f_{1} \widetilde{\wedge}_{t-s_{1}, t}^{t} f_{0}\right\rangle_{\lambda}=\left\langle f_{1}, \widetilde{\wedge}_{t-s_{1}, t}^{t} f_{0}\right\rangle_{\lambda}=\left\langle\wedge_{0, s_{1}} f_{1}, f_{0}\right\rangle_{\lambda}=\delta
$$

Propositions 2.5 and 2.6 admit a dual version.

Proposition 3.2. 1) Let $f: \bar{\Omega} \longrightarrow \mathbb{R}$ and $H:[0, t] \times \partial \Omega \longrightarrow \mathbb{R}$ two Borel bounded functions then,

$$
\begin{gathered}
E_{\lambda}\left[f\left(X_{t}^{0}\right)\right]=\int_{\Omega} f(x) \lambda(d x), \\
E_{\lambda}\left[\int_{0}^{t} H\left(r, X_{r}^{0}\right) d A_{r}^{0}\right]=\frac{1}{2} \int_{0}^{t}\left(\int_{\partial \Omega} H(r, x) d x\right) d r, \\
E_{\lambda}\left[\exp \left(\theta A_{t}^{0}\right)\right]<\infty, \quad \text { for any } \theta>0, \\
\lim _{u \rightarrow 0_{+}} \sup _{\substack{x \in \bar{\Omega} \\
0 \leq t \leq k}} E\left[A_{u}^{t, x}\right]=0, \quad(c f .(2.26)) .
\end{gathered}
$$

We omit the proof of the Proposition 3.2.

Before stating the analog of Proposition 2.1, let us introduce $\left(\mathcal{H}_{u}^{t}\right)_{0 \leq u \leq t}$ the natural filtration generated by $\left(X_{t-u} ; 0 \leq u \leq t\right)$

$$
\underline{\mathcal{H}}_{u}^{t}=\sigma\left(X_{v} ; t-u \leq v \leq t\right), \quad \mathcal{H}_{u}^{t}=\bigcap_{v<u} \underline{\mathcal{H}}_{v}^{t}
$$

Proposition 3.3. Suppose that $t>0$ and $\omega$ is a solution of (1.8).

1) a) For any $h: \bar{\Omega} \longrightarrow \mathbb{R}$ Borel bounded function,

$$
\begin{aligned}
& \left(h\left(X_{t}^{0}\right) \omega\left(t-s \wedge \xi, X^{0}(t-s \wedge \xi)\right)\right. \\
& \left.\quad \cdot \exp \left(\int_{t-s \wedge \xi}^{t} \varphi_{0}\left(r, X_{r}^{0}\right) d A_{r}\right) ; 0 \leq s \leq t\right)
\end{aligned}
$$

is a $\left(P_{\lambda}, \mathcal{H}^{t}\right)$ continuous local martingale, where $\varphi_{c}$ is defined by (2.7) and,

$(3.18) \xi=\inf \left\{s \leq t: \omega\left(t-s, X^{0}(t-s)\right)=0\right.$ and $\left.X^{0}(t-s) \in \partial \Omega\right\} \wedge t$. 
b) If $c>C_{t, \Omega}\left(C_{t, \Omega}\right.$ is defined by (2.10)),

$$
\begin{aligned}
& \left(h\left(X_{t}\right)\left(\omega\left(t-s, X^{0}(t-s)\right)+c\right)\right. \\
& \left.\quad \cdot \exp \left(\int_{t-s}^{t} \varphi_{c}\left(r, X_{r}^{0}\right) d A_{r}^{0}\right) ; 0 \leq s \leq t\right)
\end{aligned}
$$

is a $\left(P_{\lambda}, \mathcal{H}^{t}\right)$ square integrable continuous martingale.

2) The tangential component of the velocity of $X^{0}$ vanishes on the boundary.

Let $\rho$ be a velocity test function, then

$$
\frac{1}{h} E_{\lambda}\left[\rho\left(X_{T+h}^{0}\right)-\rho\left(X_{T}^{0}\right) \mid \mathcal{H}_{T}^{t}\right] \underset{h \rightarrow 0_{+}}{\stackrel{\text { a.s. }}{\rightarrow}} 0,
$$

where $T$ is the $\mathcal{H}^{t}$-stopping time,

$$
T=\inf \left\{s \leq t: X^{0}(t-s) \in \partial \Omega\right\} \wedge t .
$$

Proof. For simplicity we write $X_{t}$ instead of $X_{t}^{0}$. We set, for any $c \geq 0$

$$
\begin{aligned}
& Z_{c}(s)=h\left(X_{t}\right)\left(\omega\left(t-s, X_{t-s}\right)+c\right) \\
& \cdot \exp \left(\int_{t-s}^{t} \varphi_{c}\left(r, X_{r}\right) d A_{r}\right), \quad 0 \leq s \leq t, \\
& \xi_{n}=\inf \left\{s \leq t: \omega(t-s) X(t-s) \leq \frac{1}{n}\right. \\
& \text { and } X(t-s) \in \partial \Omega\} \wedge t, \quad n \in \mathbb{N} .
\end{aligned}
$$

$\left\{\xi_{n}\right\}_{n \geq 1}$ is an increasing sequence of $\mathcal{H}^{t}$-stopping times converging, as $n$ goes to infinity, to $\xi$.

Let $0 \leq u_{0}<u_{1} \leq t, 0 \leq s_{0}<s_{1}<\cdots<s_{n} \leq u_{0}, \Gamma_{0}, \Gamma_{1}, \ldots, \Gamma_{n}$ Borel subsets of $\mathbb{R}$ and

$$
A=\left\{X_{t-s_{0}} \in \Gamma_{0}, X_{t-s_{1}} \in \Gamma_{1}, \ldots, X_{t-s_{n}} \in \Gamma_{n}\right\} .
$$

Using the duality property (3.7) we obtain

$$
E_{\lambda}\left[Z_{c}\left(u_{1} \wedge \xi_{n}\right) \mathbf{1}_{A}\right]=E_{\lambda}\left[\widetilde{Z}_{c}^{t}\left(u_{1} \wedge \widetilde{\xi}_{n}\right) \mathbf{1}_{\widetilde{A}}\right]
$$


with $\widetilde{A}=\left\{\widetilde{X}_{s_{0}}^{t} \in \Gamma_{0}, \widetilde{X}_{s_{1}}^{t} \in \Gamma_{1}, \ldots, \widetilde{X}_{s_{n}}^{t} \in \Gamma_{n}\right\}$. Recall that $\widetilde{Z}_{c}^{t}$ and $\widetilde{\xi}_{n}$ were introduced in (2.8), respectively (2.14).

We apply 1) a) of Proposition 2.1

$$
E_{\lambda}\left[\widetilde{Z}_{c}^{t}\left(u_{1} \wedge \widetilde{\xi}_{n}\right) \mathbf{1}_{\widetilde{A}}\right]=E_{\lambda}\left[\widetilde{Z}_{c}^{t}\left(u_{0} \wedge \widetilde{\xi}_{n}\right) \mathbf{1}_{\widetilde{A}}\right]
$$

The duality property implies (3.17).

A similar approach and (2.18) estimate prove (3.19). As for (3.20) we mimic the proof of (2.11).

Recall that $\left\{\xi_{n}\right\}_{n \geq 1}$ is the increasing sequence of stopping time, converging to $\xi$. Using the stopping theorem and (3.12) we obtain:

Corollary 3.4. For any bounded Borel function $h, t>0$ and $n \geq 1$, we have

$$
\begin{aligned}
&\langle h, \omega(t, \cdot)\rangle_{\lambda}= \int_{\Omega} h(x) \omega(t, x) d \lambda(x) \\
&= E_{\lambda}\left[h\left(X_{t}^{0}\right) \omega\left(t-\xi_{n}, X^{0}\left(t-\xi_{n}\right)\right)\right. \\
&\left.\cdot \exp \left(\int_{t-\xi_{n}}^{t} \varphi_{0}\left(t, X_{r}^{0}\right) d A_{r}^{0}\right)\right], \\
&\langle h,(\omega(t, \cdot)+c)\rangle_{\lambda}=\int_{\Omega} h(x)(\omega(t, x)+c) \lambda(d x) \\
&=E_{\lambda}\left[\omega_{0}\left(X_{0}^{0}\right) h\left(X_{t}^{0}\right) \exp \left(\int_{0}^{t} \varphi_{c}\left(r, X_{r}^{0}\right) d A_{r}^{0}\right)\right] .
\end{aligned}
$$

REMARK 3.5. 1) A priori we are not allowed to drop $\xi_{n}$ in (3.24) since we do not know if

$$
\begin{aligned}
& \left(Z_{0}(s) ; 0 \leq s \leq t\right) \\
= & \left(h\left(X_{t}^{0}\right) \omega\left(t-s, X^{0}(t-s)\right) \exp \left(\int_{t-s}^{t} \varphi_{0}\left(r, X_{r}^{0}\right) d A_{r}^{0}\right) ; 0 \leq s \leq t\right)
\end{aligned}
$$

is a $\left(P_{\lambda}, \mathcal{H}^{t}\right)$-martingale.

We note that if $\omega_{0}$ is analytic, $\omega$ is also an analytic function defined on $\mathbb{R}_{+} \times \bar{\Omega}([\mathrm{Ko}])$. Therefore $\left\{(t, x) \in \mathbb{R}_{+} \times \partial \Omega: \omega(t, x)=0\right\}$ is a finite union of $C^{\infty}$ curves. $X$ being a nice diffusion process, it does not visit 
this set: for any $t>0$, almost surely, $\omega\left(t, X_{r}\right) \neq 0$ for all $r \in[0, t]$. Hence,

$$
\int_{0}^{r}\left|\varphi_{0}\left(r, X_{r}\right)\right| d A_{r}<+\infty, \quad \text { almost sure }
$$

We conjecture that

$$
\langle h, \omega(t, \cdot)\rangle_{\lambda}=E_{\lambda}\left[\omega_{0}\left(X_{0}^{0}\right) h\left(X_{t}^{0}\right) \exp \left(\int_{0}^{t} \varphi_{0}\left(r, X_{r}^{0}\right) d A_{r}\right)\right] .
$$

2) Following the convention (3.6) we rather write the former identity

$$
\langle h, \omega(t, \cdot)\rangle_{\lambda}=E_{\omega_{0} \cdot \lambda}\left[h\left(X_{t}^{0}\right) \exp \left(\int_{0}^{t} \varphi_{0}\left(r, X_{r}^{0}\right) d A_{r}\right)\right] .
$$

Recall (see Lemma 1.1) that $u$ can be expressed through $\omega$ via an integral

$$
u(t, x)=K \omega(t, x)=\int_{\Omega} \nabla_{x}^{\perp} G(x, z) \omega(t, z) d z,
$$

$G$ being the Green function of $\Delta$ on $\Omega$. Therefore we have the formal expression of $u$

$$
u(t, x)=E_{\omega_{0} \cdot \lambda}\left[\nabla_{x}^{\perp} G\left(x, X_{t}\right) \exp \left(\int_{0}^{t} \varphi_{0}\left(r, X_{r}\right) d A_{r}\right)\right] .
$$

We point out that the right-hand side of (3.29) is a double integral with respect the probability measure $\lambda \otimes P$. It seems difficult to check that this integral is convergent in some sense.

Let us define the stochastic differential system (S.N.S*.) based on $x$ :

(S.N.S*.1) Suppose $x \in \bar{\Omega}$. Let us consider the following reflected stochastic differential equation in $\bar{\Omega}$,

$$
\begin{aligned}
& X_{s}^{0, x}=x+B_{s}+\int_{0}^{s} K \omega\left(r, X_{r}^{0, x}\right) d r s \geq 0, \\
&-\int_{0}^{s} n\left(X_{r}^{0, x}\right) d A_{r}^{0, x}, \quad s \geq 0 .
\end{aligned}
$$


$K \omega$ being defined by (1.2).

(S.N.S*.2) For any $h: \bar{\Omega} \longrightarrow \mathbb{R}$ Borel bounded function,

$$
\left(\left(h\left(X_{t}^{0}\right) \omega\left(t-s \wedge \xi, X^{0}(t-s \wedge \xi)\right) \exp \left(\int_{t-s \wedge \xi}^{t} \varphi_{0}\left(r, X_{r}^{0}\right) d A_{r}^{0}\right) ; 0 \leq s \leq t\right)\right.
$$

is a $\left(P_{\lambda}, \mathcal{H}^{t}\right)$ continuous local martingale, where $\varphi_{c}$ is defined by $(2.7)$ and $\xi$ by $(3.18)$.

(S.N.S*.3) Let $\rho$ be a velocity test function,

$$
\frac{1}{h} E_{\lambda}\left[\rho\left(X_{T+h}^{0}\right)-\rho\left(X_{T}^{0}\right) \mid \mathcal{H}_{T}^{t}\right] \underset{h \rightarrow 0_{+}}{\stackrel{\text { a.s. }}{\longrightarrow}} 0
$$

where $T$ is defined by (3.21).

We are able to state a second stochastic system equivalent to the (N.S.) one.

Proposition 3.6. Suppose that (S.N.S*.) $(=($ S.N.S*.1) + (S.N.S*.2) + (S.N.S*.3)) has a solution $\left(\omega,\left\{\left(X_{s}^{t, x} ; 0 \leq s \leq t\right),\left(A_{s}^{t, x} ; 0 \leq s \leq\right.\right.\right.$ $t) ; t \geq 0, x \in \bar{\Omega}\} \omega$ being a smooth function; then $u=K \omega$ is a solution of the (N.S.) equation. Conversly if $u=K \omega$ solves the (N.S.) equation, then the (S.N.S*.) system has a unique solution.

Proof. It is a direct consequence of duality (Proposition 3.1) and Proposition 2.3.

\section{Branching particle system associated with the Navier- Stokes equation.}

1) Heuristically (see the Remark 3.5) $\omega(t, \cdot)$ can be interpreted as a density function

$$
\langle h, \omega(t, \cdot)\rangle_{\lambda}=E_{\omega_{0} \cdot \lambda}\left[h\left(X_{t}\right) \exp \left(\int_{0}^{t} \varphi_{0}\left(r, X_{r}\right) d A_{r}\right)\right] .
$$

If the sign of $\varphi_{0}$ is constant and negative, $\omega(t, \cdot)$ is truly the density of $X_{t}$, starting with initial "distribution" $\omega_{0} \cdot d \lambda$, and killed with the multiplicative functional

$$
\left(\exp \int_{0}^{t} \varphi_{0}\left(r, X_{r}\right) d A_{r} ; t \geq 0\right)
$$


Here $\varphi_{0}$ is not negative. To take into account the sign of $\varphi_{0}$, a branching particule system $Y$ is very adapted. We keep in mind that $\varphi_{0}<0$ (respectively $\varphi_{0}>0$ ) corresponds to disappearing (respectively creation) of mass.

More precisely we know that $Y$ takes its values in the set of finite linear combinations of Dirac measures

$$
Y_{t}=\sum_{i=1}^{N_{t}} \alpha_{t}^{i} \delta_{Y_{t}^{i}}
$$

where $\alpha_{t}^{i}$ belongs to $\mathbb{N}, Y_{t}^{i}$ is an element of $\bar{\Omega}$.

Then if $h: \bar{\Omega} \longrightarrow \mathbb{R}$ is a bounded Borel function, we set

$$
\left\langle h, Y_{t}\right\rangle=\int_{\bar{\Omega}} h(x) d Y_{t}(x)=\sum_{i=1}^{N_{t}} \alpha_{t}^{i} h\left(Y_{t}^{i}\right) .
$$

The aim of this section is the construction of a branching particle system $Y$ such that

$$
\langle h, \omega(t, \cdot)\rangle_{\lambda}=E\left[\left\langle h, Y_{t}\right\rangle\right] .
$$

$\omega$ appears as the mean value of the density of particles $\left(Y_{t}^{i}\right)$ associated with $Y$.

2) We follow the introduction of branching particle system given by Dynkin $[D]$ and we adapt directly the general definitions to our context. Such a system is based on three ingredients:

a) a Markov process $\left(\left(X_{s}^{x, t} ; s \geq 0\right), x \in \bar{\Omega}, t \geq 0\right)$ coming from $(3.4)$,

b) a positive continuous additive functional $C$ of $X$,

c) an offspring distribution $p=\left(p_{n}(t, x) ; t \geq 0, x \in \bar{\Omega}\right)_{n \geq 1}$ on $\mathbb{N}$, indexed by $\mathbb{R}_{+} \times \Omega$ : for any $n, p_{n}$ is a non negative Borel function and

$$
\sum_{n \geq 0} p_{n}(t, x)=1
$$

We denote by $\alpha$ the generating function associated with $\left(p_{n}(t, x) ; t \geq\right.$ $0, x \in \bar{\Omega})$

$$
\alpha(t, x, u)=\sum_{n \geq 0} p_{n}(t, x) u^{n}, \quad u \in[0,1]
$$


It is supposed, that

$$
\beta(t, x)=\sum_{n \geq 0} n p_{n}(t, x) \text { is bounded } .
$$

The description of the branching particle system $Y$ with parameters $X$, $C$ and $p$ (we note for simplicity $Y=(X, C, p)$ ) is easy to understand. Suppose that the system starts with on particle located at $x \in \bar{\Omega}$. We choose $\xi_{1}$ an exponential random variable with parameter one, independent of $X$. The dynamic of the initial particle is given by $X$ up to the first branching time $U_{1}=\inf \left\{s \geq 0: C_{s}>\xi_{1}\right\}$. At time $U_{1}$, the particle dies and a random number $N_{U_{1}}$ of new particles spring from the ancestor particle, according to $p$. The conditional distribution of $N_{U_{1}}$ given the past up to time $U_{1}$, is $\left(p_{n}\left(U_{1}, X_{U_{1}}\right) ; n \geq 0\right)$. The $N_{U_{1}}$ particles move independently off each other, as $X$, up to a second branching stopping time. A new branching occurs, and so on.

3) Let $X, C$ and $p$ be the parameters of $Y$.

We denote by $\left(Y_{s}^{t, x} ; s \geq 0\right)$ the branching particle system starting with one particle at $x \in \bar{\Omega}$, with dynamic $\left(X_{s}^{t, x} ; s \geq 0\right)$ and offspring distribution $\left(p_{n}(t+s, x) ; s \geq 0, x \in \bar{\Omega}\right)_{n \geq 1}$ and

$$
W(t, x ; s)=E\left[\exp \left(-\left\langle h, Y_{s}^{t, x}\right\rangle\right)\right]
$$

for any $(s, x) \in \mathbb{R}_{+} \times \bar{\Omega}, h: \bar{\Omega} \longrightarrow \mathbb{R}_{+}$Borel positive bounded function.

$W$ solves the basic equation (see $[\mathrm{D},(1.5)]$ )

$$
\begin{aligned}
W(t, x ; s)= & E\left[\int_{0}^{s} \bar{\alpha}\left(t+r, X_{r}^{t, x}, W\left(u, X_{r}^{t, x} ; s-r\right)\right) d C_{r}\right] \\
& +E\left[\exp -h\left(X_{s}^{t, x}\right)\right], \quad s \geq 0,
\end{aligned}
$$

where

$$
\bar{\alpha}(t, x ; u)=\alpha(t, x ; u)-u=\sum_{n \geq 0} p_{n}(t, x) u^{n}-u
$$

where $t \geq 0, x \in \bar{\Omega}, u \in[0,1]$.

4) We are interested by

$$
v(t, x ; s)=E\left[\left\langle h, Y_{s}^{t, x}\right\rangle\right], \quad s \geq 0,
$$


$h$ being a positive and bounded Borel function.

Obviously $\left\langle h, Y_{s}^{t, x}\right\rangle \geq 0$, however we do not know if this positive random variable has a finite expectation. We are interested by branching processes $Y$ such that,

$$
\sup _{x, 0 \leq s \leq t} E\left[\left\langle h, Y_{s}^{t, x}\right\rangle\right]<+\infty
$$

for all $h$ Borel positive bounded functions. It is clear that previous assumption is equivalent to

$$
\sup _{x, 0 \leq s \leq t} E\left[\left\langle 1, Y_{s}^{t, x}\right\rangle\right]<+\infty
$$

We remark that $\left\langle 1, Y_{s}^{t, x}\right\rangle$ is the number of particles still living at time $s$.

Proposition 4.1. Let $Y$ be a branching process with parameters $(X, C, p)$. We suppose that (4.11) holds. Then the function $v$ defined by (4.10) solves the "integral" equation

$$
\begin{aligned}
v(t, x ; s)= & E\left[\int_{0}^{s}\left(\beta\left(t+r, X_{r}^{t, x}\right)-1\right) v\left(t, X_{r}^{t, x} ; s-r\right) d C_{r}\right] \\
& +E\left[h\left(X_{s}^{t, x}\right)\right]
\end{aligned}
$$

where $h$ is a positive and bounded Borel function and $\beta$ defined by

$$
\beta(s, x)=\sum_{k \geq 0} k p_{k}(s, x)<+\infty
$$

and verify

$$
\sup _{x, s \leq t} \beta(s, x)<+\infty
$$

Proof of Proposition 4.1. Since $t>0$ and $x \in \bar{\Omega}$ are supposed to be fixed we write $X$ (respectively $Y, v$ ) instead of $X^{t, x}$ (respectively $\left.Y^{t, x}, v(t, x ; \cdot)\right)$. Let $a$ be a positive number, and $W$ the function defined by $(4.7)$, where $h$ is replaced by $a h$

$$
W(s)=E\left[\exp -a\left\langle h, Y_{s}\right\rangle\right] .
$$


Applying the dominated convergence theorem and (4.11), we have,

$$
\left.\frac{\partial}{\partial a} W(s)\right|_{a=0}=-E\left[\left\langle h, Y_{s}\right\rangle\right]
$$

On the other hand, (4.15) and (4.11) imply,

$$
\begin{gathered}
\sup _{x, u, r \leq s}\left|\frac{\partial \bar{\alpha}}{\partial u}(t+r, x, u)\right| \leq 1+\sup _{x, r \leq t+s} \beta(r, x)<+\infty, \\
\sup _{x, r \leq s}\left|\frac{\partial W}{\partial a}(r)\right| \leq \sup _{x, r \leq s} E\left[\left\langle h, Y_{s}\right\rangle\right]<+\infty .
\end{gathered}
$$

Since $W$ solves (4.8), we are allowed to take the partial derivative with respect to $a$, in (4.8). If we choose $a=0$, we obtain immediately (4.13).

Lemma 4.2. Let $(X, C, p)$ be the parameters of a branching process $Y$. We suppose,

$$
E^{x, t}\left[\exp \left(\theta C_{s}\right)\right]<+\infty,
$$

for any $x \in \bar{\Omega}, s, t \geq 0$, and $\theta>0$, and

$$
p_{k}(s, x)=0, \quad \text { if } k \geq 3 .
$$

Then (4.11) holds.

Proof of Lemma 4.2. 1) Let $\tau$ be the right inverse of $C: \tau_{t}=\inf \{s>$ $\left.0, C_{s}>t\right\}$. By a changing of time,

$$
\left\langle 1, Y_{\tau_{s}}^{t, x}\right\rangle=\left\langle 1, \bar{Y}_{s}^{t, x}\right\rangle, \quad s \geq 0
$$

where $\bar{Y}$ is the branching process associated with $\left(X_{\tau}, t, p\right)$.

Assumption (4.17) tells us that

$$
\left\langle 1, \bar{Y}_{s}^{t, x}\right\rangle \leq \rho_{s}
$$

Where $\left(\rho_{s} ; s \geq 0\right)$ is the Yule process (each particle lives an exponential time and then splits into two particles). It is well know ([AN, p. 109]) that $\rho_{s}$ is geometrically distributed with parameter $e^{b s}, b$ being a positive constant

$$
P\left(\rho_{s}=k+1\right)=e^{-b s}\left(1-e^{-b s}\right)^{k}, \quad k \geq 0 .
$$


Therefore

$$
E\left(\rho_{s}^{2}\right) \leq 2 e^{2 b s}
$$

2) We set $N_{s}=\left\langle\mathbf{1}, Y_{\tau_{s}}^{t, x}\right\rangle$.

We have,

$$
E\left(N_{s}\right)=\sum_{n \geq 0} E\left[N_{s} 1_{\left\{\tau_{n} \leq s<\tau_{n+1}\right\}}\right]
$$

Moreover,

$E\left(N_{s} \mathbf{1}_{\left\{\tau_{n} \leq s \leq \tau_{n+1}\right\}}\right] \leq E\left(N_{\tau_{n+1}} \mathbf{1}_{\left\{s \geq \tau_{n}\right\}}\right) \leq\left(E\left[\left(N_{\tau_{n+1}}\right)^{2}\right] P\left(s \geq \tau_{n}\right)\right)^{1 / 2}$

On the one hand, (4.18), (4.19) and (4.20) imply

$$
E\left[\left(N_{\tau_{n+1}}\right)^{2}\right] \leq E\left[\rho_{n+1}^{2}\right] \leq 2 e^{2 b(n+1)} .
$$

On the other hand,

$$
P\left(s \geq \tau_{n}\right)=P\left(C_{s} \geq n\right)=P\left(e^{\theta C_{s}} \geq e^{\theta n}\right) \leq e^{-\theta n} E\left[e^{\theta C_{S}}\right] \leq e^{-\theta n} E\left(e^{\theta C t}\right),
$$

$\theta$ being a positive number.

Let us take $\theta=3 b$, making use of (4.16), we obtain

$$
P\left(s \geq \tau_{n}\right) \leq C e^{-3 b n} .
$$

As a result

$$
\sup _{x, s \leq t} E\left(N_{s}\right) \leq C^{\prime}\left(\sum_{n \geq 0} e^{-b(n / 2)}\right)<\infty,
$$

(4.12) (or (4.11)) follows immediately.

We now investigate uniqueness in (4.13).

Lemma 4.3. Let $t>0$, and $h$ be a bounded and positive Borel function. We suppose that (4.15) holds and

$$
\lim _{s \rightarrow 0_{+}} \sup _{x \in \bar{\Omega}} E^{x, t}\left(C_{s}\right)=0 .
$$

Then there exists at most one bounded function $v$ solving (4.13). 
Proof of Lemma 4.3. Suppose that $v_{1}$ and $v_{2}$ are two bounded solutions of (4.13). We set $v=v_{1}-v_{2}$. Then

$$
v(t, x ; s)=E\left[\int_{0}^{s}\left(\beta\left(t+r, X_{r}^{t, x}\right)-1\right) v\left(t, X_{r}^{t, r}, s-r\right) d C_{r}\right]
$$

(4.15) implies that,

$$
\lambda:=1+\sup _{x, s \leq t} \beta(s, x)<+\infty .
$$

Consequently, (4.21) implies that, there exists $0<t_{0} \leq t$ such that

$$
\sup _{x \in \bar{\Omega}} E^{x, t}\left(C_{s}\right) \leq \frac{1}{2 \lambda}, \quad \text { for any } s \leq t_{0} .
$$

We come back to $(4.22)$

$$
\sup _{x, s \leq t_{o}}|v(t, x ; s)| \leq \lambda\left(\sup _{x, s \leq t_{0}}|v(t, x ; s)|\right) \frac{1}{2 \lambda} .
$$

Since $v$ is bounded, the former inequality says that $v(t, x ; \cdot)$ vanishes on $\left[0, t_{0}\right]$.

By the same way, $v(t, x ; \cdot)=0$ on $\left[t_{0}, 2 t_{0}\right] \cap[0, t]$.

This shows by induction that $v(x, t ; \cdot)=0$ on $[0, t]$.

5) Let $\left(\left(X_{s}^{t, x} ; s \geq 0\right), x \in \bar{\Omega}, t \geq 0\right)$ be a diffusion process, taking its values in $\bar{\Omega}$, and $C$ be a continuous, non-decreasing additive functional, vanishing at 0 verifying (4.16) and (4.21).

We introduce a new additive functional based on a Borel function $a: \mathbb{R}_{+} \times \bar{\Omega} \longrightarrow \mathbb{R}$

$$
C^{(a)}(s)=\int_{0}^{s}\left|a\left(t+r, X_{r}^{t, x}\right)\right| d C_{r}, \quad s \geq 0 .
$$

It is supposed

$$
\sup _{x, s \leq t}|a(x, s)|<+\infty
$$

Consequently,

$$
C^{(a)}(s) \leq \lambda_{t} C_{s}, \quad \text { for all } s \leq t
$$


where $\lambda_{t}$ is a positive constant, independent of $x$. We define the offspring distribution $p^{(a)}$ associated with $a$

$$
\begin{array}{ll}
p_{k}^{(a)}(t, x)=0, & \text { if } k \neq 0 \text { or } k \neq 2, \\
p_{0}^{(a)}(t, x)=1, & \text { if } a(t, x)<0, \\
p_{2}^{(a)}(t, x)=1, & \text { if } a(t, x) \geq 0 .
\end{array}
$$

In other words,

$$
p^{(a)}(t, x)=\mathbf{1}_{\{a(t, x)<0\}} \delta_{0}+\mathbf{1}_{\{a(t, x) \geq 0\}} \delta_{2} .
$$

Theorem 4.4. Let $Y$ be the branching process, $Y=\left(X, C^{(a)}, p^{(a)}\right)$. We suppose that (4.16) and (4.21) hold. Then for any $t \geq 0, x \in \bar{\Omega}, h$ Borel, positive, bounded function

$$
E\left[h\left(X_{s}^{t, x}\right) \exp \left(\int_{0}^{s} a\left(t+r, X_{r}^{t, x}\right) d C_{r}\right)\right]=E\left[\left\langle h, Y_{s}^{t, x}\right\rangle\right] .
$$

Proof of Theorem 4.4. 1) Let $t \geq 0, x \in \bar{\Omega}$ and $h \geq 0$ be fixed. We set

$$
\widetilde{v}(t, x ; s)=E\left[h\left(X_{s}^{t, x}\right) \exp \left(\int_{0}^{s} a\left(t+r, X_{r}^{t, x}\right) d C_{r}\right)\right]
$$

and

$$
v(t, x ; s)=E\left[\left\langle h, Y_{s}^{t, x}\right\rangle\right] .
$$

(4.16) and (4.25) imply that $C^{(a)}$ also verifie (4.16).

Obviously (4.17) is realized, therefore Lemma 4.2 tells us that $Y$ verifies (4.11). Applying Proposition 4.1, $v$ solves

$$
\begin{aligned}
& v(t, x ; s)=E\left[\int_{0}^{s}\left(\beta^{(a)}\left(t+r, X_{r}^{t, x}\right)-1\right)\right. \\
& \left.\cdot v\left(t, X_{r}^{t, x} ; s-r\right) d C_{r}^{(a)}\right]+E\left[h\left(X_{s}^{t, x}\right)\right], \\
& \beta^{(a)}(s, x)=\sum_{k \geq 0} k p_{k}^{(a)}(s, x) .
\end{aligned}
$$


but

$$
\begin{gathered}
\beta^{(a)}(s, x)-1=2 \cdot \mathbf{1}_{\{a(s, x) \geq 0\}}-1=\operatorname{sgn}(a(s, x)), \\
d C_{r}^{(a)}=\left|a\left(t+r, X_{r}^{t, x}\right)\right| d C_{r},
\end{gathered}
$$

(4.31) can be reduced as follows,

$$
\begin{aligned}
v(t, x ; s)= & E\left[\int_{0}^{s} a\left(t+r, X_{r}^{t, x}\right) v\left(t, X_{r}^{t, x} ; s-r\right) d C_{r}\right] \\
& +E\left[h\left(X_{s}^{t, x}\right)\right] .
\end{aligned}
$$

2) Suppose that $\widetilde{v}$ solves (4.32).

We have already remarked that $Y$ verifies (4.11), then $\widetilde{v}$ is bounded.

$C^{(a)}$ has the property (4.21) (it is an easy consequence of (4.25) and (4.21)).

Applying Lemma 4.3, we can conclude that $v=\widetilde{v}$. This means that (4.28) is verified.

3) We have to prove that $\widetilde{v}$ solves (4.32).

We set

$$
\rho=E\left[\int_{o}^{s} a\left(t+r, X_{r}^{t, x}\right) \widetilde{v}\left(t, X_{r}^{t, x}, s-r\right) d C_{r}\right] .
$$

Using the definition of $\widetilde{v}$, and Markovian notations,

$$
\begin{aligned}
\rho=E\left[\int_{0}^{s} a\left(t+r, X_{r}^{t, x}\right)\right. \\
\left.\quad \cdot E^{t, X_{r}^{t, x}}\left[h\left(X_{s-r}\right) \exp \left(\int_{0}^{s-r} a\left(t+u, X_{u}\right) d C_{u}\right)\right] d C_{r}\right] .
\end{aligned}
$$

A straightforward application of the Markov property gives

$$
\begin{aligned}
\rho=E\left[\int_{0}^{s} a\left(t+r, X_{r}^{t, x}\right) h\left(X_{s}^{t, x}\right)\right. & \\
& \left.\cdot\left(\exp \left(\int_{0}^{s-r} a\left(t+r+u, X_{u+r}^{t, x}\right) d C_{u+r}\right)\right) d C_{r}\right] .
\end{aligned}
$$

It is convenient to introduce the following multiplicative functional,

$$
M_{r}=\exp \left(\int_{0}^{r} a\left(t+u, X_{u}^{t, x}\right) d C_{u}\right) .
$$


We have

$$
\begin{gathered}
M_{s}=M_{r} \exp \left(\int_{r}^{s} a\left(t+u, X_{u}^{t, x}\right) d C_{u}\right) \\
=M_{r} \exp \left(\int_{0}^{s-r} a\left(t+r+u, X_{u+r}^{t, x}\right) d C_{u+r}\right), \quad r \leq s \\
d M_{r}=a\left(t+r, X_{r}^{t, x}\right) M_{r} d C_{r}
\end{gathered}
$$

Hence

$$
\begin{gathered}
\rho=E\left[M_{s} h\left(X_{s}^{t, x}\right)\left(\int_{0}^{s} \frac{1}{\left(M_{r}\right)^{2}} d M_{r}\right)\right] \\
\rho=E\left[M_{s} h\left(X_{s}^{t, x}\right)\left(1-\frac{1}{M_{s}}\right)\right]=E\left[M_{s} h\left(X_{s}^{t, x}\right)\right]-E\left[h\left(X_{s}^{t, x}\right)\right] .
\end{gathered}
$$

But $\widetilde{v}(t, x ; s)=E\left[M_{s} h\left(X_{s}^{t, x}\right)\right]$, then $\widetilde{v}$ verifies $(4.32)$.

We are now able to prove that $\omega+c$ can be interpreted as the "density" of a branching process $Y_{c}, \omega$ denoting the solution of the (N.S.) equation. We apply the Theorem 4.4. We have to define the underlying process and the functions $a, p$ and $C$.

6) Let $\left(\left(X_{s}^{t, x} ; s \geq 0\right) ; x \in \bar{\Omega}, t \geq 0\right)$ be the family of diffusions defined by (3.4).

- $c$ denotes a constant, $c>C_{t, \Omega}$. (Recall that $C_{t, \Omega}$ is defined by (2.10) and $\omega(s, x)+c>0$ for any $s \in[0, t]$ and $x \in \bar{\Omega})$.

- We define the function $a$ as follows

$$
a(s, x)=\left(\frac{\frac{\partial \omega}{\partial n}}{\omega+c}\right)(s, x) \mathbf{1}_{\{x \in \partial \Omega\}} .
$$

- $p$ is the offspring distribution based on a (cf. (4.26)),

$$
\begin{array}{ll}
p_{k}(s, x)=0, & \text { if } k \neq 0 \text { or } k \neq 2 \text { or } x \notin \partial \Omega, \\
p_{0}(t, x)=1, & \text { if } \frac{\partial \omega}{\partial n}(t, x)<0 \text { and } x \in \partial \Omega, \\
p_{2}(t, x)=1, & \text { if } \frac{\partial \omega}{\partial n}(t, x) \geq 0 \text { and } x \in \partial \Omega .
\end{array}
$$


We will say that $(s, x)$ is a annihilation (respectively creation) point of the vortex if $x \in \partial \Omega$ and $(\partial \omega / \partial n)(s, x)<0$ (respectively $(\partial \omega / \partial n)$ $(s, x) \geq 0)$.

- $C^{(a)}$ coincides with $A^{(a)}$, where $A$ is the local time process associated with $X$ (see (3.4)), namely

$$
C_{s}^{(a)}=\int_{0}^{s}\left|\frac{\partial \omega}{\partial n}\left(r, X_{r}^{t, x}\right)\right| \frac{1}{\omega\left(r, X_{r}^{t, x}\right)+c} d A_{r}^{t, x}
$$

Before stating the main result of this paper, we recall a notation (see for instance (2.30))

$$
E_{h \cdot \lambda}\left[\left\langle f, Y_{s}\right\rangle\right]=\int_{\Omega} h(x) E\left[\left\langle f, Y_{s}^{x}\right\rangle\right] \lambda(d x)
$$

where $h$ and $f$ are two Borel and positive functions, $\lambda$ is the normalized Lebesgue measure on $\Omega,\left(Y_{s}^{x} ; s \geq 0\right)$ is the branching process starting at $\delta_{x}$ associated with $X^{0, x}, C$, and $p$ ).

Theorem 4.5. Let $t>0, c>C_{t, \Omega}, \omega$ be the vorticity solution of the (1.8) system. Then

$$
E_{\left(\omega_{0}+c\right) \cdot \lambda}\left[\left\langle h, Y_{s}\right\rangle\right]=\int_{\Omega} h(x)(\omega(s, x)+c) \lambda(d x),
$$

for any $s \leq t$, and $h$ Borel and positive function.

Proof of Theorem 4.5. We apply Theorem 4.4

$$
E\left[h\left(X_{s}^{0, x}\right) M_{s}\right]=E\left[\left\langle h, Y_{s}^{0, x}\right\rangle\right], \quad s \leq t,
$$

where $h \geq 0$ and

$$
M_{s}=\exp \left(\int_{0}^{s}\left(\frac{1}{\omega+c} \frac{\partial \omega}{\partial n}\right)\left(r, X_{r}^{0, x}\right) d A_{r}^{0, x}\right) .
$$

We multiply the former equality by $\left(\omega_{0}(x)+c\right)$, we integrate with respect to $\lambda(d x), \omega_{0}+c$ being non-negative, we have

$$
E_{\left(\omega_{0}+c\right) \cdot \lambda}\left[\left\langle h, Y_{s}^{0}\right\rangle\right]=E_{\left(\omega_{0}+c\right) \cdot \lambda}\left[h\left(X_{s}^{0}\right) M_{s}\right] .
$$


Let $\gamma$ be equal to the right hand-side of the previous identity. Using duality ( $c f$. Proposition 3.1), we have

$\gamma=E_{\lambda}\left[\left(\omega_{0}+c\right)\left(X_{0}^{0}\right) h\left(X_{s}^{0}\right) M_{s}\right]=\int_{\Omega} h(x) E\left[\left(\omega_{0}+c\right)\left(\widetilde{X}_{s}^{0, x}\right) \widetilde{M}_{s}\right] \lambda(d x)$.

Obviously $\widetilde{M}_{s}=M_{s}, \widetilde{M}_{s}\left(\omega_{0}+c\right)\left(X_{s}^{x}\right)=\widetilde{Z}_{c}^{s, x}(s),\left(\widetilde{Z}_{c}^{s, x}(r), 0 \leq r \leq s\right)$ being the process defined by $(2.8)$, then

$$
\gamma=\int_{\Omega} h(x) E\left[\widetilde{Z}_{c}^{s, x}(s)\right] \lambda(d x)
$$

But $\left(\widetilde{Z}_{c}^{s, x}(r) ; 0 \leq r \leq s\right)$ is a martingale (cf. 1) b)), Proposition 2.1), therefore,

$$
E\left[\widetilde{Z}_{c}^{s, x}(s)\right]=E\left(\widetilde{Z}_{c}^{s, x}(0)\right)=\omega(s, x) .
$$

This achieves the proof of (4.38).

REMARK 4.6. We have proved,

$$
\begin{aligned}
& E_{\left(\omega_{0}+c\right) \cdot \lambda}\left[\left\langle h, Y_{s}\right\rangle\right] \\
& \quad=E_{\left(\omega_{0}+c\right) \cdot \lambda}\left[h\left(X_{s}^{0}\right) \exp \left(\int_{0}^{s} \varphi_{c}\left(r, X_{r}^{0}\right) d A_{r}\right)\right],
\end{aligned}
$$

where

$$
\varphi_{c}=\frac{1}{\omega+c} \frac{\partial \omega}{\partial n}
$$

\section{The particle algorithm associated with the branching pro- cess.}

In sections 3 and 4 , we suppose that the solution $u$ (or $\omega$ ) of the (N.S.) system is given, and then we defined two nonlinear stochastic processes $X$ and $\widetilde{X}$, and a branching process $Y$. We proved that $u$ and $\omega$ can be expressed through $X, \widetilde{X}$ and $Y$. In this nonlinear context, it is classical $[\mathrm{McK}]$ to introduce a particle algorithm having the propagation of chaos property. Our closed formulas allow us to guess the dynamic of the particle system associated with the (N.S.) equations. Unfortunately we are not able to check the convergence. We are convince that is it interesting to write it out, it will appear as a program. 
1) Let $N \geq 1$ be a fixed integer ( $N$ will go to infinity later). Recall that $u_{0}$ is the initial data in (N.S.), and $\omega_{0}=\operatorname{rot} u_{0}$.

a) $X_{0}^{1}, \ldots, X_{0}^{N}$ denote $N$ independent and equidistributed random variables taking its values in $\bar{\Omega}$ with common density

$$
\frac{1}{\int_{\Omega}\left(\omega_{0}(x)+\gamma\right) d x}\left(\omega_{0}+\gamma\right)
$$

where $\gamma$ is a constant, supposed to be large enough. In particular $\omega_{0}(x)+\gamma>0$, for all $x \in \bar{\Omega}$.

b) We define the underlying system of particles, up to the first branching time in the McKean's sens.

$X=\left(X_{t}^{i, N} ; t \geq 0 ; 1 \leq i \leq N\right)$ is the $\bar{\Omega}^{N}$-values diffusion solving the (linear) reflected stochastic differential equation

$$
\begin{aligned}
X_{t}^{i, N}= & X_{0}^{i}+B_{t}^{i}+\int_{0}^{t} u_{N}\left(s, X_{s}^{i, N}\right) d s \\
& \quad-\int_{0}^{t} n\left(X_{s}^{N}\right) d A_{s}^{N}, \quad 0 \leq i \leq N, t \geq 0, \\
A_{t}^{N}= & \int_{0}^{t} \mathbf{1}_{\left\{X_{s}^{N} \in \partial \Omega^{N}\right\}} d A_{s}^{N}, \quad t \geq 0 .
\end{aligned}
$$

$n$ denotes the outer normal of $\partial \Omega^{N},\left(B^{i} ; 1 \leq i \leq N\right)$ are $N$ independent two dimensional Brownian motions and $B_{0}^{i}=0$, independant of $\left(X_{0}^{i} ; 1 \leq i \leq N\right)$.

The function $u_{N}$ will be defined in c). It corresponds to some approximation of $u$.

c) Let $\mu_{N}$ be the empirical measure,

$$
\mu_{N}(t)=\frac{1}{N} \sum_{i=1}^{N} \delta_{X_{t}, N}
$$

Recall (Lemma 1.1) that

$$
u(t, z)=\int_{\Omega} \nabla_{z}^{\perp} G\left(z, z^{\prime}\right) \omega\left(t, z^{\prime}\right) d z^{\prime},
$$


$G$ being the Green function of $\Omega$ (see (1.3)).

If $t$ is small (lower than the first branching time) the branching process $Y_{t}$ reduces to $\delta_{X t}$, therefore $\mu_{N}(t)$ is a good candidate to approximate $\omega(t, x) d x$.

We set

$$
\begin{aligned}
\widetilde{u}_{N}(t, z) & =E\left[\int_{\Omega} \nabla_{z}^{\perp} G\left(z, z^{\prime}\right) \mu_{N}\left(t, d z^{\prime}\right)\right] \\
& =\frac{1}{N} \sum_{i=1}^{N} E\left[\nabla_{z}^{\perp} G\left(z, X_{t}^{i, N}\right)\right]
\end{aligned}
$$

Unfortunately $z \longrightarrow \nabla_{z}^{\perp} G\left(z, z^{\prime}\right)$ has a singularity at $z=z^{\prime}$, therefore we regularize $\widetilde{u}_{N}$, by replacing $\widetilde{u}_{N}$ by $\widetilde{u}_{N} * V_{N}$, where $V_{N}(z) d z$ converges (in $\mathbb{R}^{2}$ ) to $\delta_{0}$ (choose for instance, $V_{N}(z)=N^{2} V(N z), V \geq 0$, $\int_{\mathbb{R}^{2}} V(x) d x=1, V$ of class $C^{\infty}$, with compact support)

$$
u_{N}(t, z)=V_{N} * \widetilde{u}_{N}(t, \cdot)(z)=\frac{1}{N} \sum_{i=1}^{N} E\left[V_{N} * \nabla^{\perp} G\left(\cdot, X_{t}^{i, N}\right)(z)\right]
$$

Hence $u_{N}$ is $C^{\infty}$, and $x \rightarrow u_{N}(t, x)$ is of class $C^{1}$, therefore the $2 N$ dimensional stochastic differential equation (5.1) has a unique and strong solution. It is meaningful to set

$$
\omega_{N}=\operatorname{rot} u_{N} .
$$

2) The first branching time.

a) Let $\xi_{1}, \xi_{2}, \ldots, \xi_{N}$ be $N$ independent, and exponential random variable (with unit parameter), independent of the previous system of particles. The first branching time $T$ is defined as follows

$$
\begin{gathered}
T=\inf \left\{T_{i}: 1 \leq i \leq N\right\} \\
T_{i}=\inf \left\{t \geq 0: \int_{0}^{t}\left(\frac{1}{\omega_{N}+\gamma}\left|\frac{\partial \omega_{N}}{\partial n}\right|\right)\left(s, X_{s}^{i}\right) d A_{s}^{N} \geq \xi_{i}\right\}
\end{gathered}
$$

b) Suppose that $T=T_{i_{0}}$. Then $X_{T}^{i_{0}, N} \in \partial \Omega$. We have the alternative

$$
\begin{aligned}
& a_{N}\left(T, X_{T}^{i_{0}, N}\right)<0, \\
& a_{N}\left(T, X_{T}^{i_{0}, N}\right)>0,
\end{aligned}
$$$$
(5.9)+
$$ 
where

$$
a_{N}(s, x)=\left(\frac{1}{\omega_{N}+\gamma} \frac{\partial \omega_{N}}{\partial n}\right)(s, x),
$$

$a_{N}$ is some approximation of $a, a$ being defined by (4.33).

i) In the negative case (5.9) - according to (4.34)-(4.35), the particle $i_{0}$ is killed at time $T=T_{i_{0}}$. The $N-1$ remaining particles start afresh

$$
\left(X_{T}^{1, N}, \ldots, X_{T}^{i_{0}-1, N}, X_{T}^{i_{0}+1, N}, \ldots, X_{T}^{N, N}\right)
$$

and move as (5.1) with drift coefficient $u_{N}^{(1)}$,

$$
u_{N}^{(1)}(t, z)=\frac{1}{N} \sum_{\substack{i=1 \\ i \neq i_{0}}}^{N} E\left[V_{N} * \nabla^{\perp} G\left(\cdot, X_{t}^{1, i, N}\right)(z)\right] .
$$

$u_{N}^{(1)}$ is associated with the empirical measure

$$
\mu_{N}^{(1)}(t, \cdot)=\frac{1}{N} \sum_{\substack{i=1 \\ i \neq i_{0}}}^{N} \delta_{X_{t}^{1, i, N}}
$$

Note that the factor of normalization is $1 / N$ and not $1 /(N-1)$.

We define as in (5.2) the second branching time and the branching dynamic.

ii) If $(5.9)+$ holds, the particle $i_{0}$ dies and has two descendants. Then the $N+1$ processes move after $T$, as previously.

After having generated $N-1$ or $N+1$ particles, a second branching time is defined by the same way.

3) Conjectures. We claim that the offsprings of one particle (for example, the first one), $Y_{N}$ coming from the former procedure converges in law, as $N$ goes to infinity, to the branching process $Y$.

Another open question is : can we take $\gamma=0$ ? In this situation we introduce in the algorithm signed particles. Particle $i$ is said positive (respectively negative) if $\omega_{0}\left(X_{0}^{i, N}\right)>0$ (respectively $\omega_{0}\left(X_{0}^{i, N}>0\right)$, the density of $X_{0}^{i, N}$ being equal to

$$
\frac{\left|\omega_{0}(x)\right|}{\int_{\Omega}\left|\omega_{0}(x)\right| d x}
$$


The sign $\xi_{0}^{i, N}$ of $X_{t}^{i, N}$ remains constant, for any time $t . \mu_{N}$ is replaced by the signed measure,

$$
\mu_{N}(t, \cdot)=\frac{1}{N} \sum_{i=1}^{N} \xi_{0}^{i, N} \delta_{X_{t}^{i, N}}
$$

When $\Omega=\mathbb{R}^{2}$, Marchioro and Pulverenti $[\mathrm{MP}]$ has introduced signed particles in order to take into account the non-positivity of $\omega$.

If $\gamma=0$, is this algorithm converging?

\section{References.}

[C.F] Constantin, P., Foias, C., Navier-Stokes equations. University of Chicago Press, Chicago, 1998.

[C.M] Chorin, A. J., Marsden, J. E., A mathematical introduction to fluid mechanics. Springer Verlag, 1990.

[D] Dynkin, E. B., Branching particle systems and superprocesses. Ann. Probab. 19 (1991), 1157-1194.

[Ka] Kahane, C., On the spatial analyticity of solutions of the Navier-Stokes equations. Arch. Rational Mech. Anal. 33 (1969), 386-405.

[Ko] Komatsu, G., Global analyticity up to tha Boundary of solutions of the Navier-Stokes equations. Comm. Pure Appl. Math. XXXIII (1980), 545-566.

[La] Ladyzhenskaya, O., The mathematical theory of viscous incompressible flow. Gordon and Breach, 1969.

[Li] Lions, J. L., Quelques méthodes de résolution des problèmes aux limites non linéaires. Dunod. Gauthier Villers, 1969.

[Li] Lions, P. L., Mathematical topics in fluids mechanics. Vol. 1. Incompressible models. Oxford Sciences Publications. Clarendon Press, 1996.

[M] Majda, A., Vorticity and mathematical theory of incompressible fluid flow. Comm. Pure Appl. Math. 39 (1986), 187-220.

[M.P] Marchioro, C., Pulvirenti, M.. Hydrodynamics in two dimensions and vortex theory. Comm. Math. Phys. 84 (1982), 483-503.

[McK] McKean, M. P., Propagation of chaos for a class of non linear parabolic equation. Lectures Series in Diff. Equations 7 (1967), 41-57.

[P] Priouret, P., Processus de Markov sur une variété à bord compacte. Ann. Inst. H. Poincaré IV (1968), 193-253.

[R] Raviart, P. A., An analysis of particle methods. Lecture Notes in Math. 1127 (1985), 243-324. Springer Verlag. 
[S.V] Stroock, D. W., Varadhan, S. R. S., Diffusion process with boundary conditions. Comm. Pure Appl. Math. XXIV (1971), 147-226.

[T] Teman, R., Navier Stokes equations: theory and numerical analysis. North Holland, 1977.

Recibido: 2 de septiembre de 1.999

Saïd Benachour, Bernard Roynette and Pierre Vallois Université Henri Poincaré Nancy 1

Département de Mathématiques

Institut Elie Cartan

BP 239

54506 Vandouvre-lès-Nancy Cedex, FRANCE benachou@iecn.u-nancy.fr roynette@iecn.u-nancy.fr vallois@iecn.u-nancy.fr 